\title{
Irinotecan/scFv co-loaded liposomes coaction on tumor cells and CAFs for enhanced colorectal cancer therapy
}

Zhaohuan $\mathrm{Li}^{1 \dagger}$, Chunxi Liư ${ }^{2 \dagger}$, Chenglei Li ${ }^{1}$, Fangqing Wang ${ }^{3}$, Jianhao Liu' ${ }^{1}$ Zengjuan Zheng ${ }^{1}$, Jingliang $\mathrm{Wu}^{3^{*}}$ and Bo Zhang ${ }^{1^{*}}$ (D)

\begin{abstract}
Background: Cancer-associated fibroblasts (CAFs), as an important component of stroma, not only supply the "soils" to promote tumor invasion and metastasis, but also form a physical barrier to hinder the penetration of therapeutic agents. Based on this, the combinational strategy that action on both tumor cells and CAFs simultaneously would be a promising approach for improving the antitumor effect.

Results: In this study, the novel multifunctional liposomes (IRI-RGD/R9-sLip) were designed, which integrated the advantages including IRI and scFv co-loading, different targets, RGD mediated active targeting, R9 promoting cell efficient permeation and lysosomal escape. As expected, IRI-RGD/R9-sLip showed enhanced cytotoxicity in different cell models, effectively increased the accumulation in tumor sites, as well as exhibited deep permeation ability both in vitro and in vivo. Notably, IRI-RGD/R9-sLip not only exhibited superior in vivo anti-tumor effect in both CAFs-free and CAFs-abundant bearing mice models, but also presented excellent anti-metastasis efficiency in lung metastasis model.
\end{abstract}

Conclusion: In a word, the novel combinational strategy by coaction on both "seeds" and "soils" of the tumor provides a new approach for cancer therapy, and the prepared liposomes could efficiently improve the antitumor effect with promising clinical application prospects.

Keywords: Colorectal cancer, Liposomes, Cancer-associated fibroblasts, Single chain fragment variable, Co-cultured cells

\footnotetext{
*Correspondence: jlwu2008@163.com; zhangbo@wfmc.edu.cn

†Zhaohuan Li and Chunxi Liu contributed equally to this work

1 School of Pharmacy, Weifang Medical University Weifang,

Shandong 261053, People's Republic of China

${ }^{3}$ School of Bioscience and Technology, Weifang Medical University,

Weifang 261053, Shandong, People's Republic of China

Full list of author information is available at the end of the article
}

(c) The Author(s) 2021. Open Access This article is licensed under a Creative Commons Attribution 4.0 International License, which permits use, sharing, adaptation, distribution and reproduction in any medium or format, as long as you give appropriate credit to the original author(s) and the source, provide a link to the Creative Commons licence, and indicate if changes were made. The images or other third party material in this article are included in the article's Creative Commons licence, unless indicated otherwise in a credit line to the material. If material is not included in the article's Creative Commons licence and your intended use is not permitted by statutory regulation or exceeds the permitted use, you will need to obtain permission directly from the copyright holder. To view a copy of this licence, visit http://creativecommons.org/licenses/by/4.0/. The Creative Commons Public Domain Dedication waiver (http://creativeco mmons.org/publicdomain/zero/1.0/) applies to the data made available in this article, unless otherwise stated in a credit line to the data. 


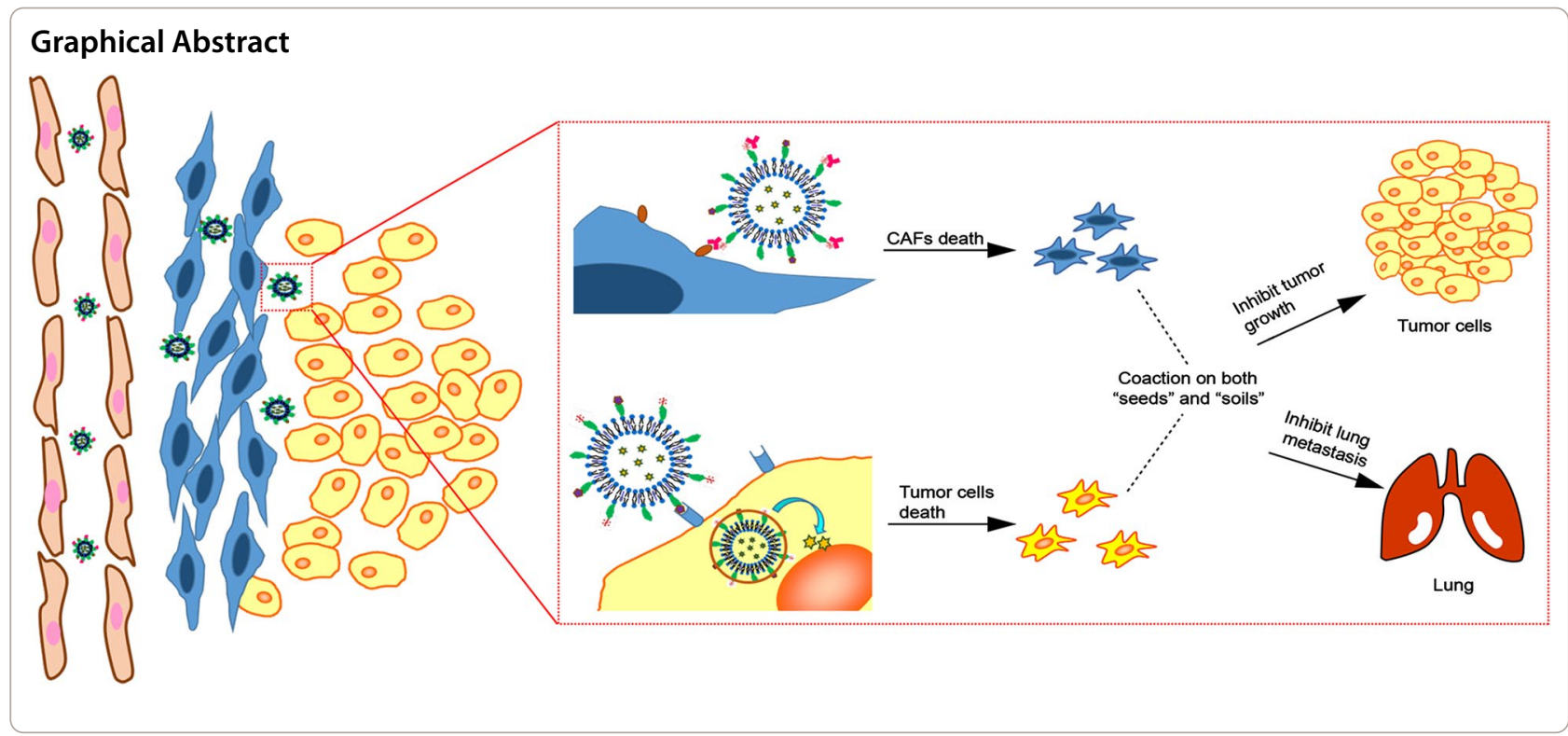

\section{Background}

Colorectal cancer (CRC) has become the third most common cancer with high morbidity and mortality $[1$, 2]. Chemotherapy is one of the important treatment manners in clinic, for example, Irinotecan (IRI), as the first-line chemotherapeutic agent for colorectal cancer, has been approved into market by many countries $[3,4]$. IRI will be metabolized into pharmacological activated 7-ethyl-10-hydroxy camptothecin (SN-38), which could inhibit topoisomerase I activity, and result in doublestranded DNA rupture [5]. However, the application of chemotherapy is far from success, which does not significantly improve patients' cure rates or long-term survival [6]. With the deep understanding in cancer, it is well recognized that tumor microenvironment (TME) plays a crucial role in supporting the growth and invasion of tumor cells. Tumor cells can be considered as the "seeds" of cancer, while TME would provide the nutrients and favorable environment as the "soils" [7-10]. Therefore, on the basis of eliminating the "seeds" by chemotherapeutics, it is necessary to further destroy the "soils" for tumor cells survival to reshape the TME and thus effectively reduce tumor metastasis and recurrence.

TME is composed of extracellular matrix and numerous of stromal cells $[11,12]$. Among that, cancer associated fibroblasts (CAFs) are the important components in tumor stroma especially in CRC, which is derived from normal fibroblasts and activated during the crosstalk with tumor cells $[13,14]$. CAFs can promote tumor genesis, metastasis, and drug resistance by secreting the cytokines and extracellular matrix proteins such as $\alpha$-smooth muscle actin $(\alpha-S M A)$ and fibroblast activator protein (FAP) [15-17]. FAP is a typeII transmembrane protein, which is highly expressed in CAFs of various cancers $[18,19]$. In recent years, FAP has attracted widespread attention as an attractive target $[20,21]$. Yu et al. designed the novel thermosensitive liposomes with FAP- $\alpha$ specific response, which not only killed tumor cells, but also destroyed stromal barrier to further promote deep tumor penetration [22]. Fan et al. developed one kind of $\mathrm{pH}$-sensitive nanoparticles, which showed little cytotoxicity to normal tissue but could kill cancer cells and stromal cells efficiently [23]. In another study by Freedman et al. bispecific T-cell engagers (BiTE) were proposed, which could specifically kill the tumor cells as well as destroy the CAFs [24]. Single chain fragment variable ( $\mathrm{scFv}$ ) is a non-covalent heterodimer that specifically binds to FAP and inhibits the function of FAP $[25,26]$. The use of FAP chimeric antibody $\mathrm{scFv}$ could eliminate the physical barrier formed by CAFs and inhibit cells metastasis. Herein, we attempt to explore whether the combination on IRI and $\mathrm{scFv}$ would be beneficial for enhanced CRC therapy by killing both tumor cells and CAFs simultaneously.

However, the combination therapy of IRI or scFv would face inevitable obstacles in many aspects. Firstly, it is hard for free drugs to produce synergistic effects due to the significant difference between small molecules and macromolecular drugs [27], accordingly, drug delivery system (DDS) is required to deliver both drugs to the tumor tissue simultaneously [28]. Secondly, after accumulated into the tumor, IRI and scFv would act on tumor cells and CAFs respectively, herein it is required to ensure each drug released and target to the acting site. Thirdly, due to the high interstitial fluid pressure in TME and the physical barrier formed by CAFs, the drug permeation into the deep tumor site will be severely impeded $[29,30]$. 


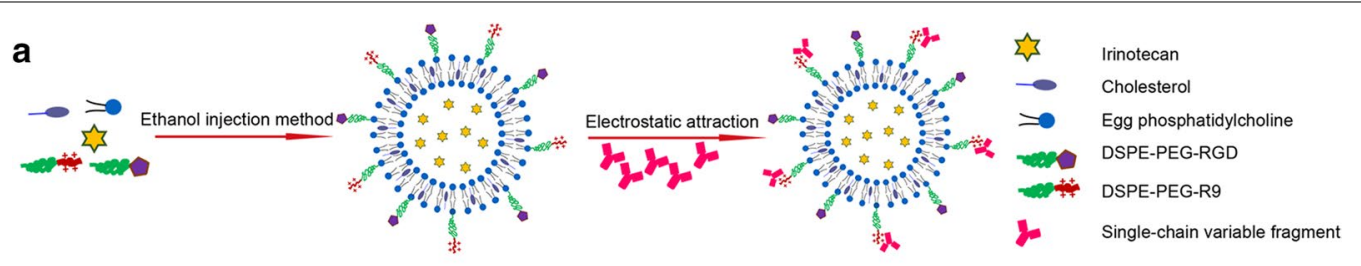

b

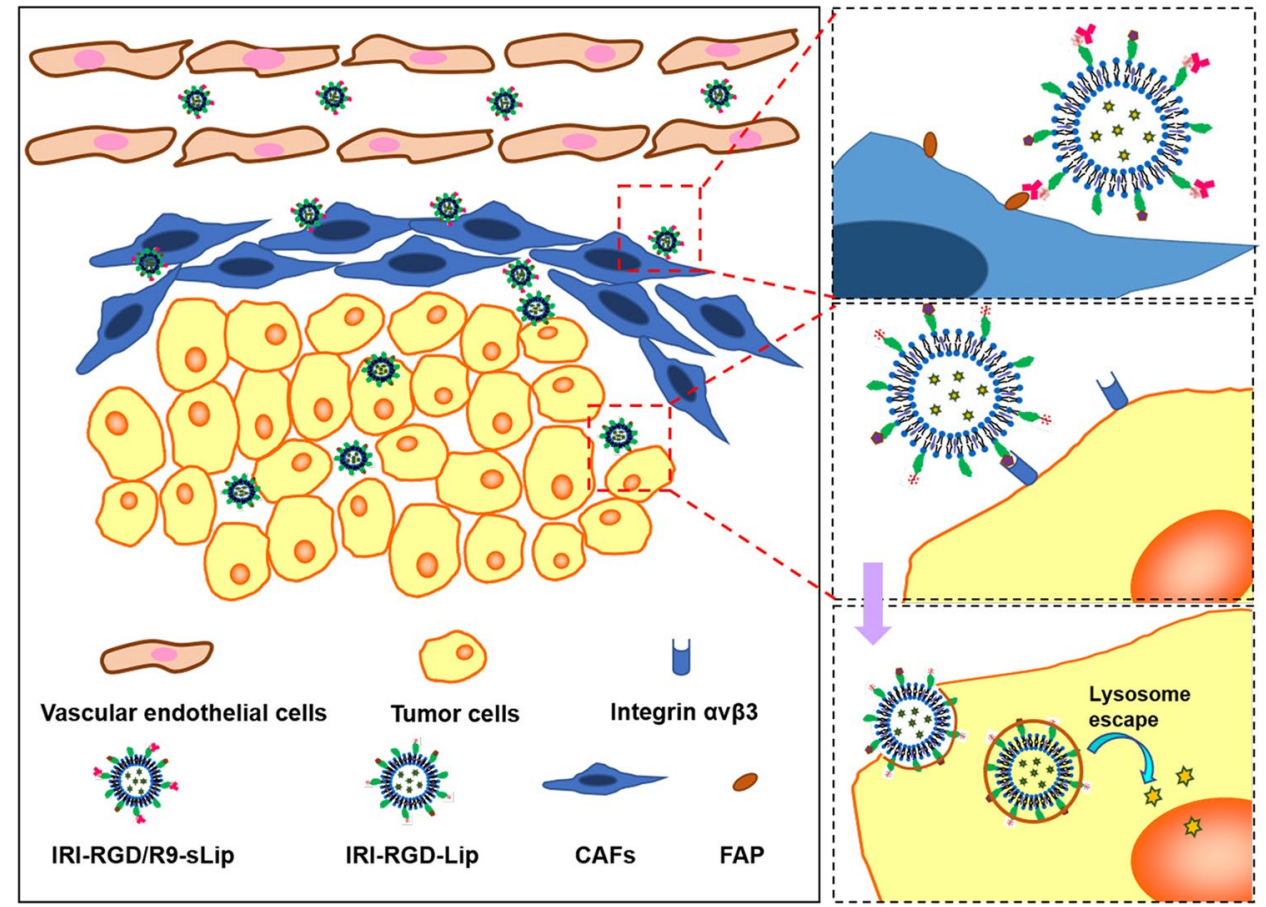

Scheme 1 Design and combinational therapy process of multi-functional liposomes (IRI-RGD/R9-sLip). a The preparation process of liposomes. $\mathbf{b}$ In vivo process of IRI-RGD/R9-sLip

Furthermore, after the drug endocytosed into the cells, it is easily degraded under the strong acidic environment of intracellular lysosomes, which makes IRI difficulty to internalize into the nucleus to exert therapeutic effects [31, 32]. Therefore, it is crucial to establish a novel DDS that could integrate the advantages in co-delivery of two drugs, responsible-release from different targets, highefficient deep permeation, and lysosomal escape to overcome the above bottlenecks.

Liposomes have nowadays become one of the promising drug delivery systems with desired safety and clinical translational prospects [33-35]. In this study, we designed one kind of novel multi-functional liposomes (IRI-RGD/R9-sLip) for the co-delivery of chemotherapeutic drug IRI and anti-FAP scFv (Scheme 1). The liposomes were further modified with RGD ligand and 9-arginine cationic peptide (R9), in which RGD can specifically recognize integrin receptors on the surface of tumor cells, while R9 has been proven to have excellent functions in cells penetration and lysosomal escape
[36-38]. As illustrated in Scheme 1, scFv was adsorbed on the surface of liposomes by electrostatic interaction with R9 and thus shielded the positive charge, which could keep stable during circulation. After accumulated into the tumor tissue, $\mathrm{scFv}$ could specifically target FAP to inhibit the proliferation of CAFs and eliminate the physical barrier of tumors. Importantly, due to the stronger binding force between FAP and its antibody ( $\mathrm{scFv}$ ), scFv was shed from the liposomes [39]. Subsequently, the uncovered cationic liposomes could further internalize into the tumor cells by RGD targeting and the excellent transmembrane ability of $\mathrm{R} 9$ can improve the deep penetration of liposomes [40, 41]. In addition, after the liposomes endocytosed into tumor cells, the amino group in R9 can further neutralize the acidic environment of the lysosomes through protonation and realize lysosomal escape of the liposomes, accordingly favoring IRI into the nucleus to inhibit the cell proliferation.

In the present work, multi-functional liposomes (IRI-RGD/R9-sLip) were prepared and evaluated for 
enhanced CRC therapy. Notably, in order to mimic the real CAFs-abundant TME, a novel co-cultured cells model was established by mixing NIH/3T3 cells with tumor cells to induce the activated CAFs. Particle sizes, zeta potentials and morphology of the liposomes were firstly characterized. Afterwards, the cellular uptake efficiency of liposomes was evaluated to verify cRGD targeting. The penetration capability of R9 was studied in vitro by establishing 3D microtumors and in vivo by frozen sections, while the lysosomal escape of R9 was confirmed by observing the distribution of liposomes in lysosomes. The in vivo antitumor effect of liposomes was assessed in two different tumor bearing mouse models, meanwhile, lung metastatic mouse model was established for antimetastasis evaluation. In summary, the novel combinational strategy by coaction on both "seeds" and "soils" of the cancer was proposed in this study, which provides a new idea for cancer therapy. It is the first attempt to prepare multifunctional liposomes that integrated co-delivery, different targets, deep permeation and lysosomal escape into one system, which could efficiently improve the antitumor effect and hold promising clinical application prospects.

\section{Materials and methods Materials}

1,2-distearoyl-sn-glycero-3-phosphoethanolamine- $\mathrm{N}$ [amin (polyethylene glycol)-2000] (DSPE-PEG $2000-\mathrm{NH}_{2}$ ) and Egg phosphatidylcholine (EPC) were obtained from AVT Pharmaceutical Technology Co., Ltd. (Shanghai, China). Lyso-Tracker Green was from Beyotime Biotechnology Co., Ltd. (Shanghai, China). Single-chain variable fragment $(\mathrm{scFv})$ was provided by Merry Bio Co., Ltd. (Nanjing, China). Cholesterol, Hoechst33258, 3-(4,5-dimethylthiazol-2-yl)-2,5-diphenyltetrazolium bromide (MTT), Agarose and 4,6-diamino-2-phenylindole (DAPI) were acquired from Solarbio (Beijing, China). Nine arginine (R9) peptide was synthesized from Shanghai Taopu Biotechnology Co., Ltd. (Shanghai, China). DSPE-PEG-cRGD was synthesized by Xi'an Ruixi Biological Technology Co., Ltd. (Xi'an, China). Irinotecan base (IRI), Doxorubicin Hydrochloride (DOX.HCl) were purchased from Meilun Biotechnology Co., Ltd. (Dalian, China). FAP1 Ab-AF5344 and alpha-SMA Ab-AF1032 were obtained from Affinity Biosciences LTD (Jiangsu, China).

\section{Cell culture and animals}

Embryonic mouse fibroblasts (NIH 3T3) and mouse CT-26 colon carcinoma cells were obtained from cell bank of Chinese Academy of Sciences (Shanghai, China). Both cells were cultured in the culture media containing $10 \%$ fetal bovine serum with addition of $1 \%$ penicillin-streptomycin. DMEM and RPMI-1640 media were selected for NIH 3T3 and CT-26 cells, respectively. The cells were cultured at $37{ }^{\circ} \mathrm{C}$ containing $5 \% \mathrm{CO}_{2}$. For the incubation of CT-26/NIH 3T3 co-cultured cells, both cells were mixed at the ratio of 1:2.

Female BALB/c mice (14-16 g) were provided by Pengyue Experimental Animal Co., Ltd. (Jinan, China). All animal experiments were conducted in accordance with the Regulations on Animal Control issued by the Ministry of Health of the People's Republic of China and the Ethical Review of Animal Experiments issued by Weifang Medical University (2017-025).

\section{Synthesis of DSPE-PEG-R9}

R9 peptide were dissolved in DMSO and activated by EDC for 10-15 min, followed by addition of NHS with stirring for $2 \mathrm{~h}$. Afterwards, DSPE-PEG2000- $\mathrm{NH}_{2}$ and a small amount of triethylamine were added to react for $24 \mathrm{~h}$ under the protection of nitrogen. The reaction solution was dialyzed for $48 \mathrm{~h}$ and then lyophilized to obtain DSPE-PEG-R9. The structure of DSPE-PEG-R9 was characterized by ${ }^{1} \mathrm{H}$-NMR. The yield and reaction percent were calculated as follows:

$$
\begin{aligned}
& \text { Yield }(\%)=\frac{\text { mass of DSPE }-P E G-R 9}{\text { mass of total }} \times 100 \% \\
& \text { Reaction percent }(\%)=\frac{\text { Area }(R 9) / \text { Number }(R 9)}{\text { Area }(\text { PEG }) / \text { Number }(P E G)} \times 100 \%
\end{aligned}
$$

In which Area $_{(\mathrm{PEG})}$ and $\mathrm{Area}_{(\mathrm{R} 9)}$ represent the peak area of "alkyl in PEG" and "guanidyl in R9", repectively. While Number $_{(\mathrm{PEG})}$ and Number $_{(\mathrm{R} 9)}$ represent the hydrogen proton numbers of "alkyl in PEG" and "guanidyl in R9", respectively.

\section{Preparation of liposomes}

Before the preparation of liposomes, DSPE-PEG-R9 was obtained by one-step amide reaction using DSPE$\mathrm{PEG}_{2000}-\mathrm{NH}_{2}$ and $\mathrm{R} 9$ peptide. IRI-RGD/R9-Lip were prepared via the following procedures. IRI, EPC, DSPEPEG-cRGD, DSPE-PEG-R9 and cholesterol were dissolved in $2 \mathrm{~mL}$ ethanol to form a lipophilic solution, in which the mole ratio of DSPE-PEG-cRGD: DSPE-PEGR9: EPC was controlled at 5:5:90. The solution were slowly injected into $5 \mathrm{~mL}$ PBS and stirred at $60{ }^{\circ} \mathrm{C}$ for $1 \mathrm{~h}$. IRI-loaded liposomes (IRI-RGD/R9-Lip) were prepared after ultrasonication and filtered through $0.45 \mu \mathrm{m}$ and $0.22 \mu \mathrm{m}$ membrane for 3 times, respectively [42]. Afterwards, scFv was added and electrostatic adsorbed on the surface of IRI-RGD/R9-Lip to obtain co-loaded liposomes (IRI-RGD/R9-sLip). 
Besides, IRI-loaded liposomes without cRGD and R9 modification (IRI-Lip) were prepared as control group. IRI-RGD-Liposomes (IRI-RGD-Lip) were also prepared with the mole ratio of DSPE-PEG-cRGD: EPC at 5:95. Additionally, doxorubicin base (DOX) was obtained after $\mathrm{DOX} \cdot \mathrm{HCl}$ was dehydrochlorinated, and DOX loaded liposomes (DOX-Lip) were prepared for fluorescent trace. All the liposomes were prepared using the same method.

\section{Characterization of liposomes}

The morphologies of IRI-RGD/R9-sLip was visualized by transmission electron microscopy (TEM). Particle sizes, polydispersity index (PDI) and zeta potentials of liposomes were determined by Malvern Zetasizer Nano ZS90. To evaluate the stability of IRI-RGD/R9-sLip, the size changes was analyzed for 14 days after the samples diluted with RPMI-1640 or PBS, respectively. Besides, after the liposomes demulsified by $10 \%$ Triton $\mathrm{X}-100$, the concentrations of IRI or DOX were measured by ultraviolet (UV) spectrophotometer at the UV absorption wavelength of $360 \mathrm{~nm}$ or $480 \mathrm{~nm}$, respectively. Encapsulation efficiency (EE\%) and drug loading (DL\%) were figured out using the equations as below:

$$
\begin{aligned}
& E E \%=\frac{W_{\text {encapsulateddrug }}}{W_{\text {totaldrug }}} \times 100 \% \\
& D L \%=\frac{W_{\text {encapsulateddrug }}}{W_{\text {liposomes }}} \times 100 \%
\end{aligned}
$$

\section{In vitro release of IRI and ScFv}

The release profiles of IRI were studied using the dialysis bag method, in which $0.5 \%$ Tween- 80 were added into PBS (pH 7.4) solution to meet the sink condition [43]. Briefly, $1 \mathrm{~mL}$ free IRI or IRI-Lip were transferred into dialysis bags $(\mathrm{MWCO}=3500)$ respectively and incubated with $30 \mathrm{~mL}$ releasing medium at $37{ }^{\circ} \mathrm{C}$ under $100 \mathrm{rpm}$ shaking. At each time interval, $2 \mathrm{~mL}$ solution was extracted and supplemented with fresh release medium of the equal volume. The drug contents were analyzed by UV spectrophotometer at the absorption wavelength of $360 \mathrm{~nm}$.

Regarding the release profiles of anti-FAP $s c F v$, in consideration only after binding to the FAP on CAFs, $\mathrm{scFv}$ would be shed from the liposomes. Herein, $300 \mu \mathrm{L}$ R9-sLip were firstly incubated with activated NIH3T3 cells, and PBS group was set as control group to eliminate the influence of cell secretions. At different time points, $150 \mu \mathrm{L}$ cell supernatant solution was extracted and diluted with $150 \mu \mathrm{L}$ PBS. The content of scFv was determined by BCA microprotein quantitative kit.

\section{In vitro cytotoxicity assay}

In vitro cytotoxicity of free IRI, free scFv, IRI-Lip, IRIRGD-Lip, IRI-RGD/R9-Lip and IRI-RGD/R9-sLip were evaluated using MTT assay. $150 \mu \mathrm{L}$ of CT-26 cells $\left(5 \times 10^{3}\right.$ cells/well) or CT-26/NIH 3T3 co-cultured cells (the ratio of CT-26 to NIH 3T3 cells at 1:2) were seeded in 96-well plates and cultured overnight [44]. Different concentrations of IRI or scFv were added respectively and incubated for $48 \mathrm{~h}$. Afterwards, $20 \mu \mathrm{L}$ of MTT $(5 \mathrm{mg} / \mathrm{mL})$ was added to each well and incubated for another $4 \mathrm{~h}$. Finally, MTT medium was replaced by $150 \mu \mathrm{L}$ of DMSO. The absorbance was measured at $490 \mathrm{~nm}$ using a microplate reader (ELX800, Bio-Tek, USA). Besides, blank liposomes were incubated with CT-26 cells or CT-26/NIH 3T3 cocultured cells for $48 \mathrm{~h}$ to evaluate their cell viability.

The normal NIH 3T3 cells can be activated by tumor cells or tumor cell supernatant to become activated $\mathrm{NIH}$ 3T3 cells (CAFs). In brief, NIH 3T3 cells alone were cultured for $8 \mathrm{~h}$ and then pre-treated with supernatant of CT-26 cells for $16 \mathrm{~h}$. After that, the cells were treated with IRI, RGD/R9-Lip and RGD/R9-sLip for $48 \mathrm{~h}$ and evaluated by MTT assay in activated NIH3T3 cells (incubated with CT-26 supernatants).

\section{Cell migration assay}

Firstly, CT-26 cells $\left(4 \times 10^{5}\right.$ cells/well $)$ or CT-26/NIH 3T3 co-cultured cells were inoculated in six-well plates. After the cells grown to $90-100 \%$, three lines were scraped from each plate with the sterile tip of a spear. Each well was washed twice with PBS and treated with different preparations. For the dosage of free IRI, IRI-Lip, IRIRGD-Lip and IRI-RGD/R9-sLip, the concentration of IRI in all the formulations were fixed at $15 \mu \mathrm{g} / \mathrm{mL}$. Inverted fluorescence microscopy was used to obtain the images at $0 \mathrm{~h}$ and $24 \mathrm{~h}$. The migration rate was evaluated by area detection methods, which was measured by ImageJ software and calculated as

$$
\text { Migrationrate }=\frac{\text { Width }_{0 h}-\text { Width }_{24 h}}{\text { Width }_{0 h}} \times 100 \%
$$

\section{Western blot analysis}

After washing cells with PBS for three times and adding cell lysis buffer, the cells were scraped off with a cell scrape and centrifuged at $14,000 \mathrm{~g}$ for $5 \mathrm{~min}$. The concentration of the supernatant was quantified by Bicinchoninic acid (BCA) kit. The separated proteins that run on SDS-PAGE gels (12\%) transferred to polyvinylidene difluoride (PVDF) membrane, and the sealant containing $5 \%$ milk was used to block the membrane for $2 \mathrm{~h}$. After the membrane washed with TBST for three times, the primary antibody was added and incubated overnight. The membrane was further washed to remove 
the unbound primary antibody and then incubated with secondary antibody at room temperature for $1 \mathrm{~h}$. Ultrasensitive ECL chemiluminescence solution was added to observe the protein bands.

\section{In vitro cellular uptake}

DOX-loaded liposomes were prepared to analyze the intracellular accumulation of drugs in substitution for IRI. CT-26 cells $\left(5 \times 10^{4}\right.$ cells/well $)$ were seeded in 24 -well plates containing round glass sheet. The DOXLip or DOX-RGD-Lip at the DOX concentration of $10 \mu \mathrm{g} / \mathrm{mL}$ were added for $1 \mathrm{~h}$. To study the effect of RGD on cells uptake efficiency, the cells were firstly incubated with free RGD solution at the concentration of $1 \mathrm{mg} / \mathrm{mL}$ for $4 \mathrm{~h}$, followed by incubation with DOX-RGD-Lip for $1 \mathrm{~h}$. Each plate was washed three times with PBS. $4 \%$ tissue fixing fluid was applied to immobilize cells and then washed away. The nuclei were stained with DAPI and washed three times with PBS. Finally, the round glass was placed on the slide, and observed using a confocal laser scanning microscope (CLSM). Besides, the cells were collected and the fluorescence intensity was quantified by flow cytometry.

\section{Endosomal escape}

To verify the endosomal escape ability of DSPE-PEG-R9, CT-26 cells $\left(2 \times 10^{5}\right.$ cells/well $)$ were seeded on confocal dish. After incubation for $24 \mathrm{~h}$, the cells were incubated with DOX-Lip or DOX-R9-Lip for $0.5 \mathrm{~h}, 1 \mathrm{~h}, 2 \mathrm{~h}$ and $4 \mathrm{~h}$. After washed with PBS, the cells were incubated with Lysotracker Green (70 nM) and Hoechst 33,258 for $30 \mathrm{~min}$. The cells were observed under CLSM. The colocalization ratio of lysosome and liposome (Mander's coefficients) was calculated by ImageJ [45, 46].

\section{D Tumor spheroids}

Co-cultured cells (ratio of CT-26 to NIH 3T3 cells was $1: 2$, among which CT-26 $2 \times 10^{3}$ cells/well) were seeded onto 96-well plates with $1 \%$ agarose. After seven days of culture, a 3D tumor model was generated. DOX-Lip, DOX-RGD-Lip and DOX-RGD/R9-Lip were incubated with tumor spheroids for $6 \mathrm{~h}$. DOX fluorescence signals at different depths were scanned by CLSM.

\section{Tumor penetration in vivo}

The tumor-bearing mice model was established by subcutaneous injection of $0.1 \mathrm{~mL}$ CT-26/NIH 3T3 cocultured cells (ratio of CT-26 to NIH 3T3 cells was 1:2, among which CT-26 $1 \times 10^{7}$ cells $/ \mathrm{mL}$ ) at the right hind legs in mice. When the tumor grew to approximately 200 $\mathrm{mm}^{3}$, DOX-Lip, DOX-RGD-Lip and DOX-RGD/R9-Lip were injected intravenously at a DOX concentration of $3 \mathrm{mg} / \mathrm{kg}$. After $12 \mathrm{~h}$, the mice were sacrificed and the tumors were fixed with $4 \%$ paraformaldehyde. The different depth sections of tumor tissues were cryo-sectioned by freezing microtome at the thickness of $10 \mu \mathrm{m}$, then the nuclei were stained with DAPI and the sections were observed by CLSM.

In addition, a 3D fluorescence imaging system was applied to further verify the penetration of R9. In briefly, CT-26/NIH 3T3 co-cultured cells tumor-bearing mice models were administrated with IR-780-Lip, IR780-RGD-Lip and IR-780-RGD/R9-Lip at the IR-780 concentration of $0.1 \mathrm{mg} / \mathrm{mL}$. At 8,12 and $24 \mathrm{~h}$ post administration, the fluorescence distribution at the tumor site was observed by 3D fluorescence imaging system.

\section{In vivo animal imaging}

BALB/c mice were subcutaneously injected with CT-26 cells $\left(1 \times 10^{7}\right.$ cells $\left./ \mathrm{mL}\right)$ or CT-26/NIH 3T3 co-cultured cells. IR-780 was selected as the imaging tracker in vivo due to its strong absorption at around $780 \mathrm{~nm}$, which was beneficial to monitor the distribution in the body in real time. After the tumors grew about $100 \mathrm{~mm}^{3}$, free IR-780, IR-780-Lip, IR-780-RGD-Lip and IR-780-RGD/R9-sLip were injected intravenously at the IR-780 concentration of $0.1 \mathrm{mg} / \mathrm{mL}$. At different time intervals post administration, the fluorescence imaging was observed using the in vivo imaging system (IVIS). $24 \mathrm{~h}$ later, the mice were sacrificed to dissect the organs and tumors for further ex vivo imaging.

Moreover, orthotopic tumor model was first attempted to further evaluate the in vivo biodistribution. BABL/c mice were anesthetized with chloral hydrate $(4 \%)$ and the abdomen was cut to expose cecum. CT-26 cells $\left(1 \times 10^{6}\right.$ cells suspended in $25 \mu \mathrm{L}$ of PBS/Matrigel, $\left.1: 1 \mathrm{v} / \mathrm{v}\right)$ were injected into the colon wall to establish orthotopic tumor model. After 10 days, free IR-780, IR-780-Lip, IR780-RGD-Lip and IR-780-RGD/R9-sLip were injected intravenously at the IR-780 concentration of $0.1 \mathrm{mg} / \mathrm{mL}$. At different time intervals post administration, the fluorescence imaging was observed using the in vivo imaging system (IVIS). $24 \mathrm{~h}$ later, the mice were sacrificed to dissect the organs and tumors for further ex vivo imaging.

\section{In vivo antitumor effects}

The antitumor effects were evaluated in both CT-26 cells $\left(1 \times 10^{7}\right.$ cells $\left./ \mathrm{mL}\right)$ and CT-26/NIH $3 \mathrm{~T} 3$ co-cultured cells tumor-bearing mice models, respectively. After the tumor grew to approximately $150 \mathrm{~mm}^{3}$, the mice were randomly divided into six groups $(n=5)$ and intravenously administrated with saline, free IRI, IRI-Lip, IRI-RGD-Lip, IRI-RGD/R9-Lip or IRI-RGD/ R9-sLip every 2 days for 14 days (IRI was $20 \mathrm{mg} / \mathrm{kg}$, $\mathrm{scFv} 0.334 \mathrm{mg} / \mathrm{kg}$ ). The body weights and the tumor size 
were recorded every 2 days. Tumor volumes (V) were calculated as $\mathrm{V}=\left(\right.$ tumor length $\times$ tumor width $\left.^{2}\right) / 2$.

After treatment, the major organs were dissected from the sacrificed mice and the tumor weights were measured. Organs and tumors were fixed with $4 \%$ paraformaldehyde. All tissues were sectioned and embedded in paraffin, for hematoxylin and eosin $(\mathrm{H} \& \mathrm{E})$ staining. Apoptosis of tumor tissues was detected by Colorimetric TUNEL Apoptosis Assay Kit. Ki-67 immunohistochemistry was performed according to the instructions to identify cell proliferation. Dewaxing and antigenic repair were performed on the sections. The tissue was blocked with bovine serum albumin (BSA). Subsequently, the expression of FAP or $\alpha$-SMA was detected according to the instructions of rabbit polymer assay system. The above tissues were observed under microscope. The percentage of positive FAP or $\alpha$-SMA area to the total area was quantified using ImageJ software.

Orthotopic tumor model was also attempted for further evaluation of in vivo antitumor effects. The mice were randomly divided into three groups $(n=3)$ : (1) saline, (2) free IRI, (3) IRI-RGD/R9-sLip. After 7 times of administration, the mice were sacrificed and their colon tissues were collected for inhibitory evaluation. Afterwards, H\&E staining was also carried out to explore the proliferative status of tumor.

\section{Evaluation of lung metastasis}

Colorectal cancer is closely associated with distant metastasis, especially to the liver and lungs. To build a lung metastasis model of colorectal cancer, intravenous injection of CT-26 cells is widely applied in many studies [47-51]. Lung metastasis model was built by intravenously injecting of $0.1 \mathrm{~mL}$ CT-26 cells $\left(1 \times 10^{7}\right.$ cells/ $\mathrm{mL}$ ) into the mice. All mice were randomly divided into 7 groups $(n=3)$ and the lungs of healthy mice served as negative control $[47,52]$. One day later, mice with lung metastases were treated with Saline, free IRI, IRI-Lip, IRI-RGD-Lip, IRI-RGD/R9-Lip or IRI-RGD/R9-sLip (IRI $20 \mathrm{mg} / \mathrm{kg}$, scFv $0.334 \mathrm{mg} / \mathrm{kg}$ ). Changes in body weight of mice were detected during the treatment. After 7 times of administration, the mice were sacrificed and their lungs were collected for $H \& E$ staining and the numbers of lung nodules were recorded.

\section{Statistical analysis}

All the data were presented as the mean \pm standard deviation (SD). The differences between two groups were evaluated by Student's t test using GraphPad Prism 8.0. $P<0.05$ was considered to be statistical significance.

\section{Results and discussion}

\section{Characterizations of liposomes}

The structures of DSPE-PEG-cRGD and DSPE-PEGR9 were verified by ${ }^{1} \mathrm{H}$-NMR. The chemical shift at 6.5-8.5 ppm was assigned to characteristic peaks of cRGD, indicating that DSPE-PEG-cRGD was successfully obtained (Additional file 1: Fig. S1a). Besides, the characteristic peaks at $7.0-8.0 \mathrm{ppm}$ was assigned to guanidine group of R9, indicating that R9 was successfully conjugated to DSPE-PEG (Additional file 1: Fig. S1b). The yield of DSPE-PEG-R9 was $42 \%$ and the reaction percent of "R9 conjugated to DSPE-PEG- $\mathrm{NH}_{2}$ " was $54 \%$.

The preparation procedure of the liposomes was illustrated in Scheme 1A. Firstly, RGD and R9-modified cationic liposomes were prepared by ethanol injection, and then $\mathrm{scFv}$ was adsorbed on the surface of the cationic liposomes by electrostatic adsorption to form the co-delivery system. The zeta potential reversal of the liposomes further proved the successful synthesis of cationic liposomes and the successful adsorption of scFv on their surface (Fig. 1c).

The average particle size, PDI, zeta potentials, EE\% and LE\% of different liposomes were shown In Table 1. The mean particle sizes were less than $150 \mathrm{~nm}$ with $\mathrm{PDI}<0.2$, indicating the uniform dispersion of the liposomes. The average particle sizes and PDI of DOX-loaded and IR780Liposomes were shown in Additional file 1: Table S1. The mean particle sizes were similar to IRI-loaded Liposomes. The zeta potential of IRI-RGD/R9-sLip was negative, which was beneficial for the liposomes to remain stable in circulation. IRI base could be readily encapsulated into the liposomes due to the hydrophobicity, with the encapsulation efficacies more than $90 \%$ in all IRI-loaded liposomes. TEM images showed regular sphericity (Fig. 1a, b). As shown in Fig. 1d, the stability of liposomes was tested in PBS or RPMI1640, and the size change was negligible within 14 days, suggesting that liposomes had good stability and fit for intravenous injection. The release behaviors of free IRI and IRI-Lip in PBS was shown in Fig. 1e. The release of free IRI showed obvious burst release, and its release exceeded $80 \%$ over $4 \mathrm{~h}$. However, the release of IRI from IRI-Lip was significantly slower, which was conducive to the sustained release of drugs $(P<0.01)$. For $\mathrm{scFv}$, it could be rapidly released from R9-sLip with the release amount nearly reached $100 \%$ in $2 \mathrm{~h}$ (Additional file 1: Fig. S6), indicating that the bind of $\mathrm{scFv}$ to FAP can trigger the $\mathrm{scFv}$ shedding off from R9-sLip.

\section{In vitro cytotoxicity}

Cell viability of blank liposomes (RGD-Lip or RGD/ R9-Lip) were first evaluated in both CT-26 cells and CT-26/NIH3T3 co-cultured cells, respectively. There 

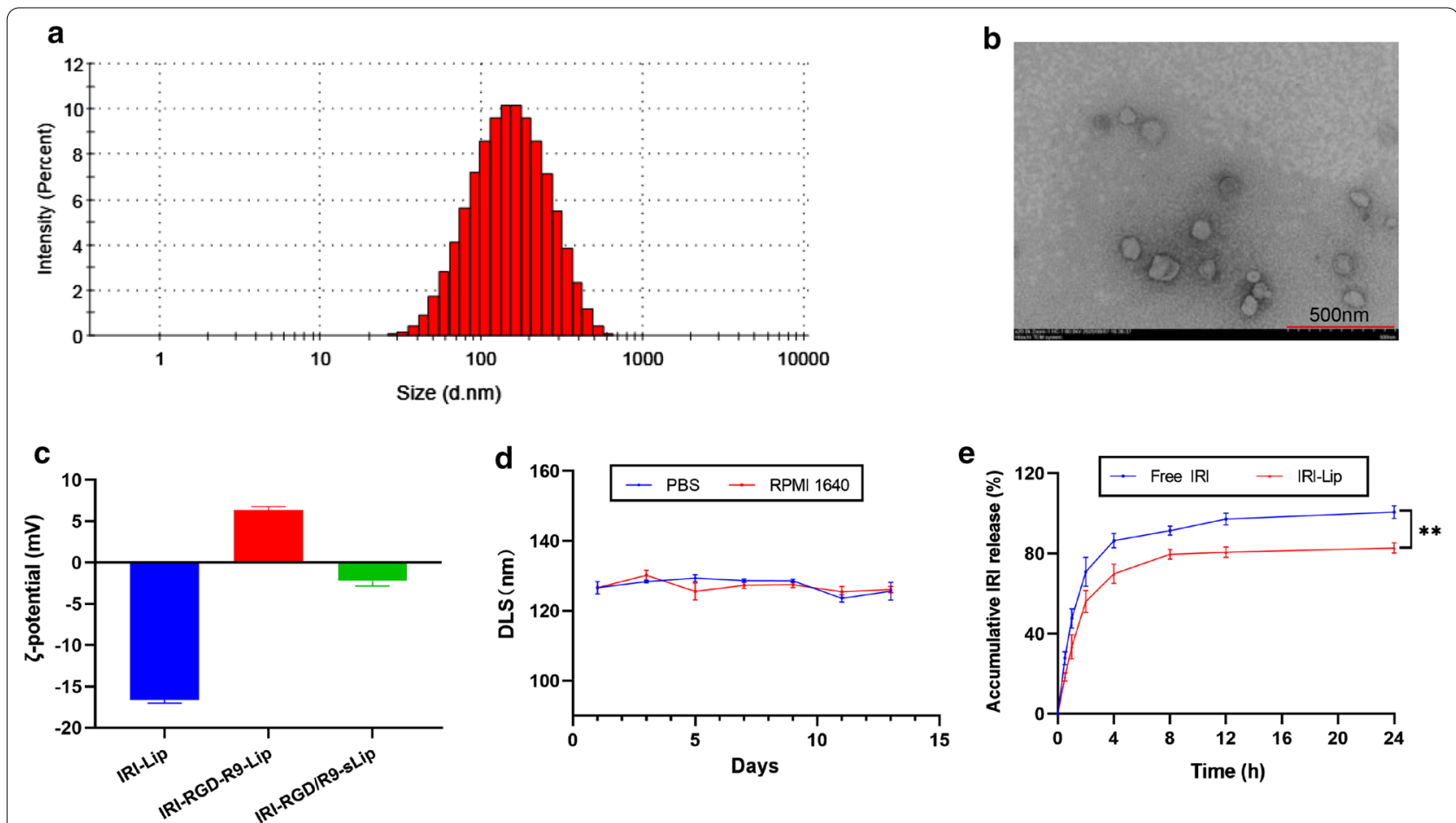

b

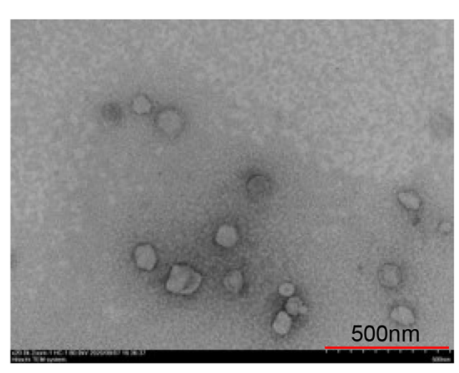

in

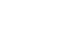

Fig. 1 Characterization of liposomes. a Representative particle size distribution and $\mathbf{b}$ TEM image of IRI-RGD/R9-sLip. c Zeta-potential changes in various formulations. $\mathbf{d}$ The particle size changes of IRI-RGD/R9-SLip in PBS or RPMI 1640 medium within 14 days. e In vitro release profiles of Free IRI or IRI-Lip. ${ }^{* *} P<0.01$

Table 1 Characterizations of the liposomes

\begin{tabular}{llllrr}
\hline & DLS $(\mathbf{n m})$ & PDI & Zeta potential $(\mathbf{m V})$ & EE (\%) & LE (\%) \\
\hline IRI-Lip & $138.3 \pm 2.0$ & $0.13 \pm 0.03$ & $-16.7 \pm 0.3$ & $90.4 \pm 2.0$ & $8.7 \pm 1.3$ \\
IRI-RGD-Lip & $141.7 \pm 0.6$ & $0.11 \pm 0.02$ & $-16.5 \pm 2.0$ & $97.4 \pm 3.3$ & $9.9 \pm 0.3$ \\
IRI-RGD/R9-Lip & $125.1 \pm 2.8$ & $0.16 \pm 0.02$ & $+6.3 \pm 0.3$ & $100.2 \pm 5.9$ & $8.9 \pm 0.5$ \\
IRI-RGD/R9-sLip & $126.8 \pm 2.6$ & $0.19 \pm 0.02$ & $-3.3 \pm 0.4$ & $98.0 \pm 2.0$ & $8.7 \pm 0.1$ \\
\hline
\end{tabular}

was no significant cytotoxicity after RGD-Lip or RGD/ R9-Lip incubation, in which the cell viabilities were above 85\% (Additional file 1: Fig. S2a, b). These results suggested that the blank liposomes had good safety profiles. Besides, the cytotoxicity of RGD/R9-sLip, RGD/ R9-Lip and free IRI were also evaluated by MTT assay in activated NIH3T3 cells (incubated with CT-26 supernatants). RGD/R9-Lip showed no significant toxicity, while RGD/R9-sLip had enhanced cytotoxicity than RGD/ R9-Lip (Additional file 1: Fig. S3). These results indicated that $\mathrm{scFv}$ could inhibit the proliferation of CAFs. The cytotoxicity of irinotecan (IRI) in activated NIH3T3 cells was also evaluated. Additionally, the cells showed concentration dependent survival-rate after IRI treatment, indicating that IRI could also kill CAFs (Additional file 1: 


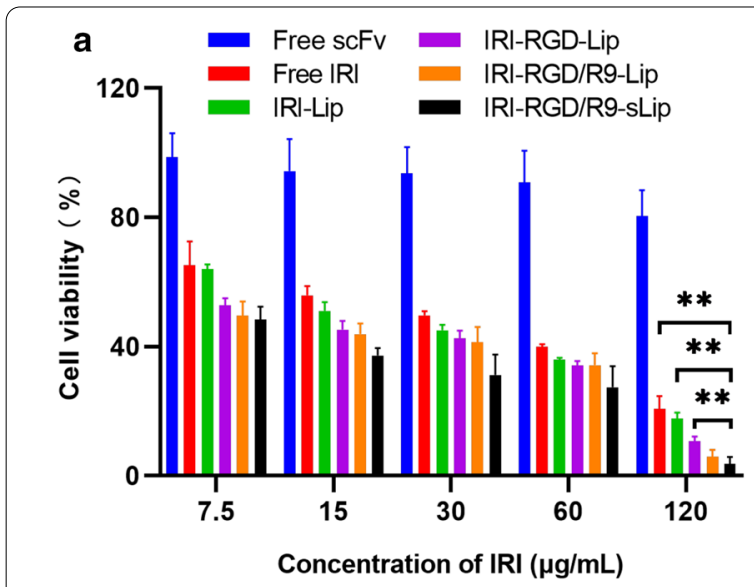

C
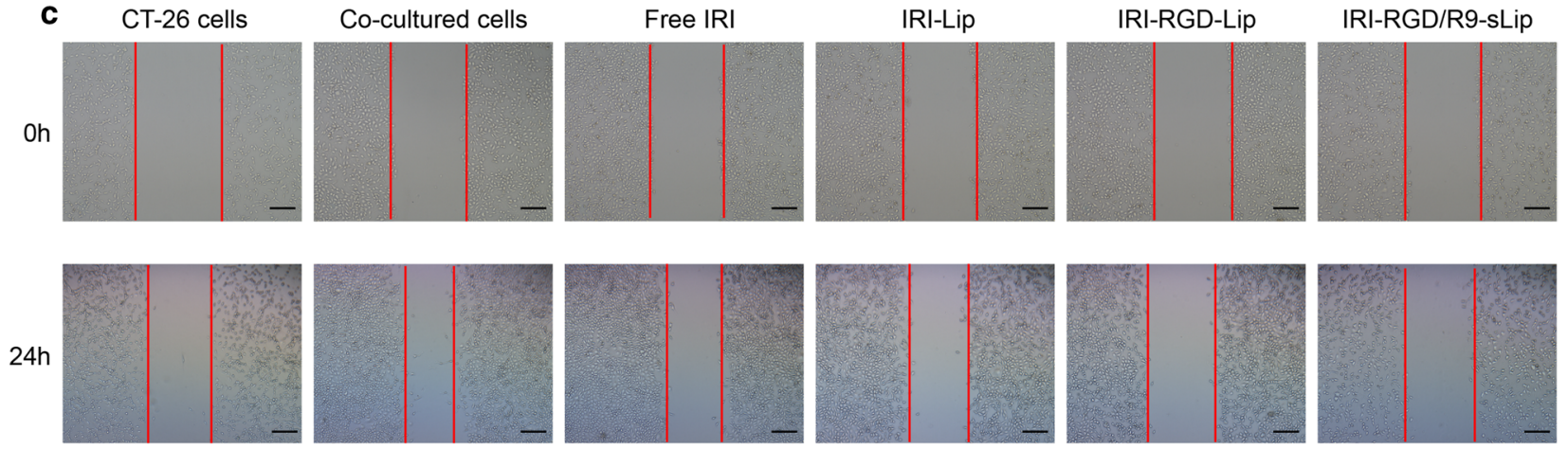

d

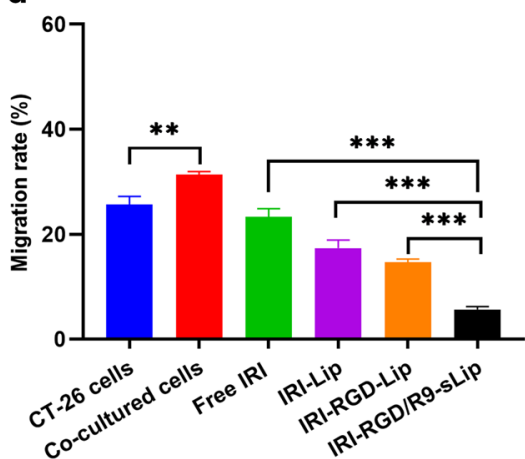

e
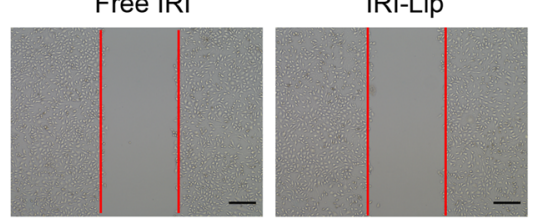

IRI-RGD-Lip

IRI-RGD/R9-sLip
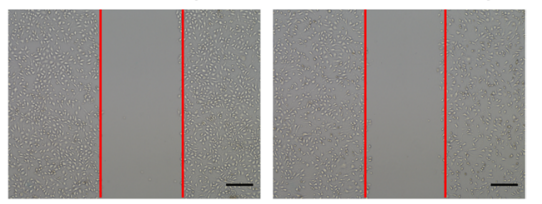

\section{f}

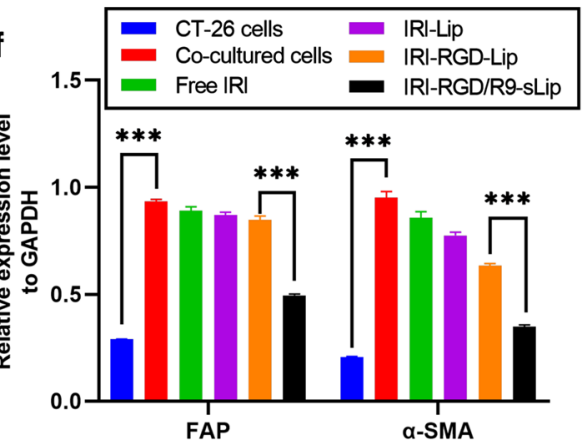

Fig. 2 Analysis of cell proliferation and migration. Viability of $\mathbf{a}$ CT-26 cells and $\mathbf{b}$ co-cultured cells (ratio of CT-26 to NIH 3T3 cells was 1:2) after incubation with different formulations at different concentrations for $48 \mathrm{~h}$. c Migration experiment after different formulations treatment for $24 \mathrm{~h}$. Scale bar, $100 \mu \mathrm{m}$. d Cell migration percentage. e Western blot analysis of FAP and a-SMA expression levels and $\mathbf{f}$ quantification data. ${ }^{* *} P<0.01$, ${ }^{* * *} P<0.001$

varying degrees reduced inhibitory effect compared to that in CT-26 cells. Particularly, compared with IRIRGD/R9-Lip, IRI-RGD/R9-sLip showed less cell viability with significant difference (Fig. 2b), which revealed that the addition of $\mathrm{scFv}$ could killing the CAFs and thus improved the cytotoxicity. In summary, these results suggested that CT-26 cells were more sensitive to chemotherapeutic agent, while the co-cultured cells showed increased tolerance to single chemotherapy. On the other hand, combination of IRI and scFv may reduce cell drug resistance by killing CAFs, and thus exhibited strong cytotoxicity.

\section{Wound healing study}

CT-26 cells were set as the control group to evaluate the migration ability of cells in comparison to the co-cultured system. As shown in the Fig. 2c, d, the cell migration rate in CT-26/NIH 3T3 co-cultured cells was obviously 
increased than that in CT-26 cells, demonstrating that CAFs promoted cell migration. After treated with different formulations, cell migration ability showed different degrees of inhibition. Among that, the combination of IRI and scFv significantly inhibited cells migration and showed strongest anti-metastasis effect, which indicated that $\mathrm{scFv}$ could reduce cell migration rate by inhibiting CAFs.

FAP and $\alpha$-SMA are the important biomarkers of CAFs with specifically up-regulated expression. Herein, the expression levels of FAP and $\alpha$-SMA were detected by western blotting to verify the activation of CAFs in co-cultured cells. As shown in Fig. 2e, f, both FAP and $\alpha$-SMA had weak expression in CT-26 cells, while obviously increased in co-cultured cells, suggesting that NIH 3T3 co-incubated with CT-26 cells could activate CAFs. Furthermore, after treatment by IRI, the expression of FAP and $\alpha$-SMA decreased in different degrees. Compared with other formulations, the expression of FAP and $\alpha$-SMA was obviously decreased after treatment by
IRI-RGD/R9-sLip. These results indicated that the combination therapy could effectively inhibit the functions of CAFs, which was beneficial for inhibition of tumor metastasis.

\section{In vitro cellular uptake}

CLSM and flow cytometry were performed to assess RGD mediated cellular uptake efficiency. Bule and red fluorescence represented nucleus and DOX, respectively. As shown in Fig. 3a, DOX-RGD-Lip group showed stronger red fluorescence than the DOX-Lip treatment group, indicating that RGD binds to integrin receptors of cells surface, thereby improving the targeting of liposomes. Furthermore, competitive inhibition test was performed to verify the enhanced uptake ability of RGD. The red fluorescence intensity decreased after excessive free RGD treatment in advance. This might be due to the saturation of cells surface receptors by free RGD, leading to reduced uptake efficiency of DOX-RGD-Lip. Similarly, the quantitative results in flow cytometry also

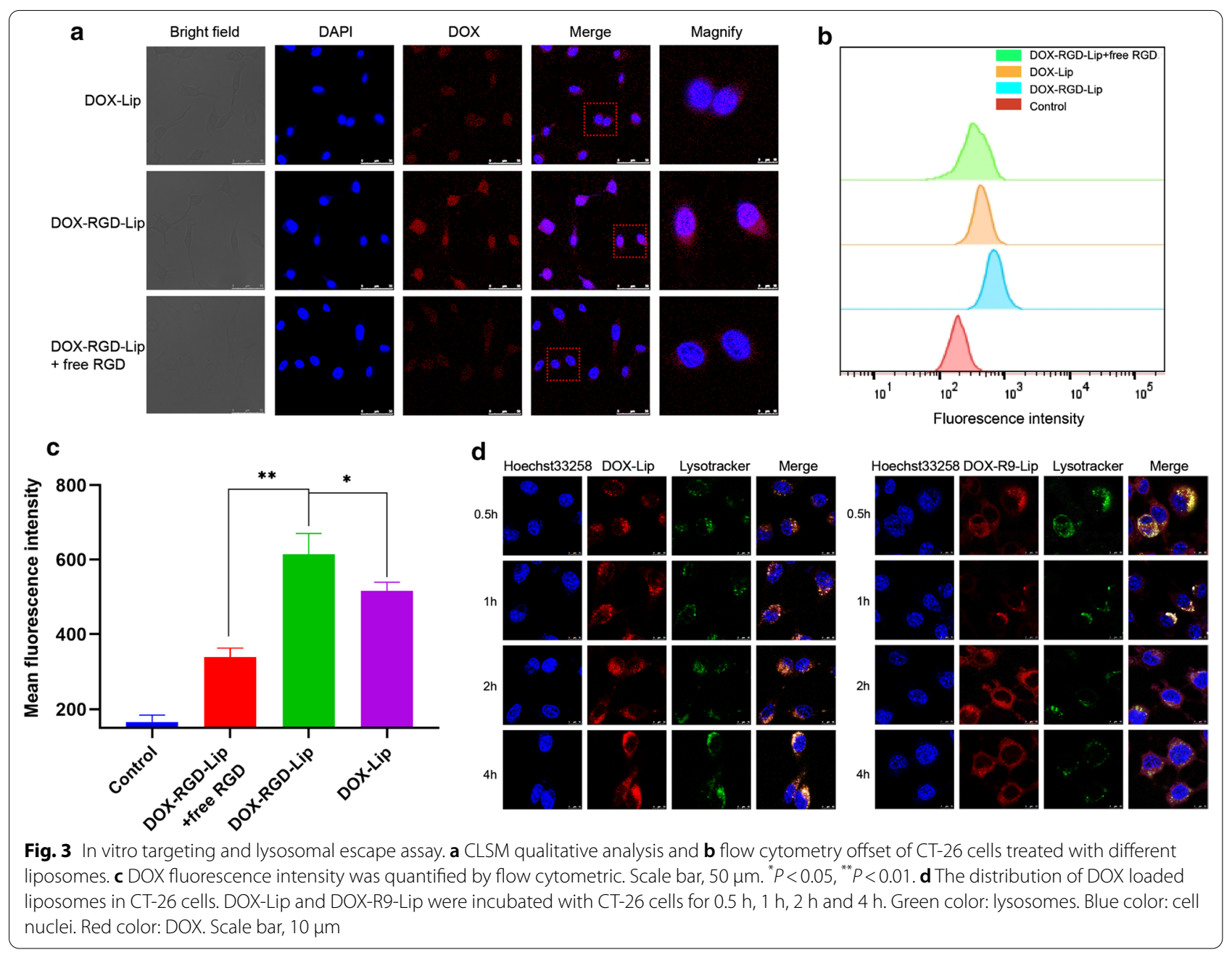


proved that the fluorescence intensity of DOX in the DOX-RGD-Lip group was 1.2-fold higher than that of the DOX-Lip group and 1.8-fold higher than that of the DOX-RGD-Lip group treated with excessive free RGD in advance (Fig. 3b, c). The above results indicated that RGD-mediated endocytosis could effectively improve cell internalization.

\section{Endosomal escape}

The lysosomal escape function of R9 peptide were verified by co-incubating DOX-R9-Lip with cells for different hours. Yellow spots represented the co-localization of DOX red fluorescence and lysosomal green fluorescence. After incubation for $0.5 \mathrm{~h}$ and $1 \mathrm{~h}$, yellow spots with high fluorescence intensity were observed in the cytoplasm, indicating that liposomes were endocytosed into the lysosomes. While at $2 \mathrm{~h}$, red fluorescence and green fluorescence were partially separated. At $4 \mathrm{~h}$, only a small amount of red fluorescence overlapped with green fluorescence, indicating that liposomes were successfully escaped from lysosomes. In contrast, in the control group (DOX-Lip), even at $4 \mathrm{~h}$, strong overlapped yellow fluorescence was observed (Fig. 3d). These results demonstrated that R9 modifies liposomes could help the drugs escape from lysosomes. Moreover, the colocalization ratio of lysosome and liposome (Mander's coefficients) was calculated and illustrated in Additional file 1: Fig. S5, the percent of DOX-R9-Lip in lysosomes decreased with time increasing, meanwhile, the percent of DOX-R9-Lip in lysosomes is much lower than DOXLip in lysosomes $(\mathrm{p}<0.01)$. All these results suggested that R9-modified liposomes would be beneficial for lysosome escape.

\section{In vitro and in vivo tumor penetration}

A novel in vitro 3D tumor sphere model constructed by co-cultured CT-26/NIH 3T3 cells were established to verify the penetration ability of R9-modified liposomes in vitro. DOX-Lip was used as a negative control and the red fluorescence represented DOX. As shown in Fig. 4a, compared with DOX-Lip group, DOX-RGD-Lip group showed enhanced permeability due to RGD targeting ability, while the fluorescence intensity increases of DOX-RGD/R9-Lip group at each depth than DOXRGD-Lip, which was attributed to the R9 strong membrane penetration ability.

To further investigate the permeability of DOXRGD/R9-Lip in vivo, subcutaneous tumor-bearing mice were administrated with DOX-Lip, DOX-RGDLip and DOX-RGD/R9-Lip by tail vein injection. As shown in Fig. 4b, DOX-Lip group and DOX-RGD-Lip group were distributed on the tumor surface, while the DOX-RGD/R9-Lip group could penetrate into deeper areas, and strong red fluorescence could be seen even at $1500 \mu \mathrm{m}$ depth. In addition, the fluorescence intensity was quantified, in which DOX-RGD/ R9-Lip at each depth was much higher than DOXLip and DOX-RGD/R9-Lip with significant differences (Fig. 4c). These results suggested that R9 could improve the penetration ability of liposomes and thus deliver the drug into deep tumor, which would be beneficial for efficiently killing the tumor cells.

In addition, the in vivo permeability of R9 was also further verified using a 3D imaging system. Weak fluorescence in IR-780-Lip indicated the poor penetration ability of normal liposomes, while enhanced permeability observed in IR-780-RGD-Lip group due to RGD active-targeting ability. Notably, IR-780-RGD/R9-Lip showed strong fluorescence at all the time points, suggesting that R9 could improve the penetration ability of liposomes (Fig. 4d). To exhibit the in vivo 3D imaging more visualized, a representive video file was attached in the Additional file 2.

\section{In vivo biodistribution study}

In vivo biological distribution assay was performed to verify the distribution behaviors of liposomes in both CT-26 bearing mice model and CT-26/NIH 3T3 bearing mice model, respectively. In CT-26 bearing mice model (Fig. 5a-c), there were more tumor accumulation in all the liposomal formulations than free IR-780, which could be attributed to the EPR effect. Besides, the fluorescence intensity in free IR-780 group declined at $24 \mathrm{~h}$, while strong signals sustained in the liposomal groups. Compared to the other liposomal formulations, enhanced fluorescence signals were observed at tumor sites in mice after treatment with IR-780-RGD-R9-sLip. It might be due to that after eliminating the barriers of CAFs by scFv, the liposomes could be more easily permeate and target into the deep tumor cells, herein increased tumor accumulation were observed. Furthermore, ex vivo imaging was carried out and quantified at $24 \mathrm{~h}$ post injection. The quantitative results revealed that IR-780-RGD/R9-sLip had higher fluorescence intensity than other formulations, due to the EPR effect and the dual targeting effect mediated by RGD and scFv. Similar results were also observed in co-cultured cells tumor-bearing mice as shown in Fig. $5 \mathrm{~d}-\mathrm{f}$, in which IR780-RGD/R9-sLip had enhanced tumor accumulation than the other groups.

In vivo distribution behaviors of liposomes were further monitored in orthotopic tumor model. Similar results were also observed in orthotopic tumor model as shown in Additional file 1: Fig. S7. Weak fluorescence was observed after administration with free IR-780, 


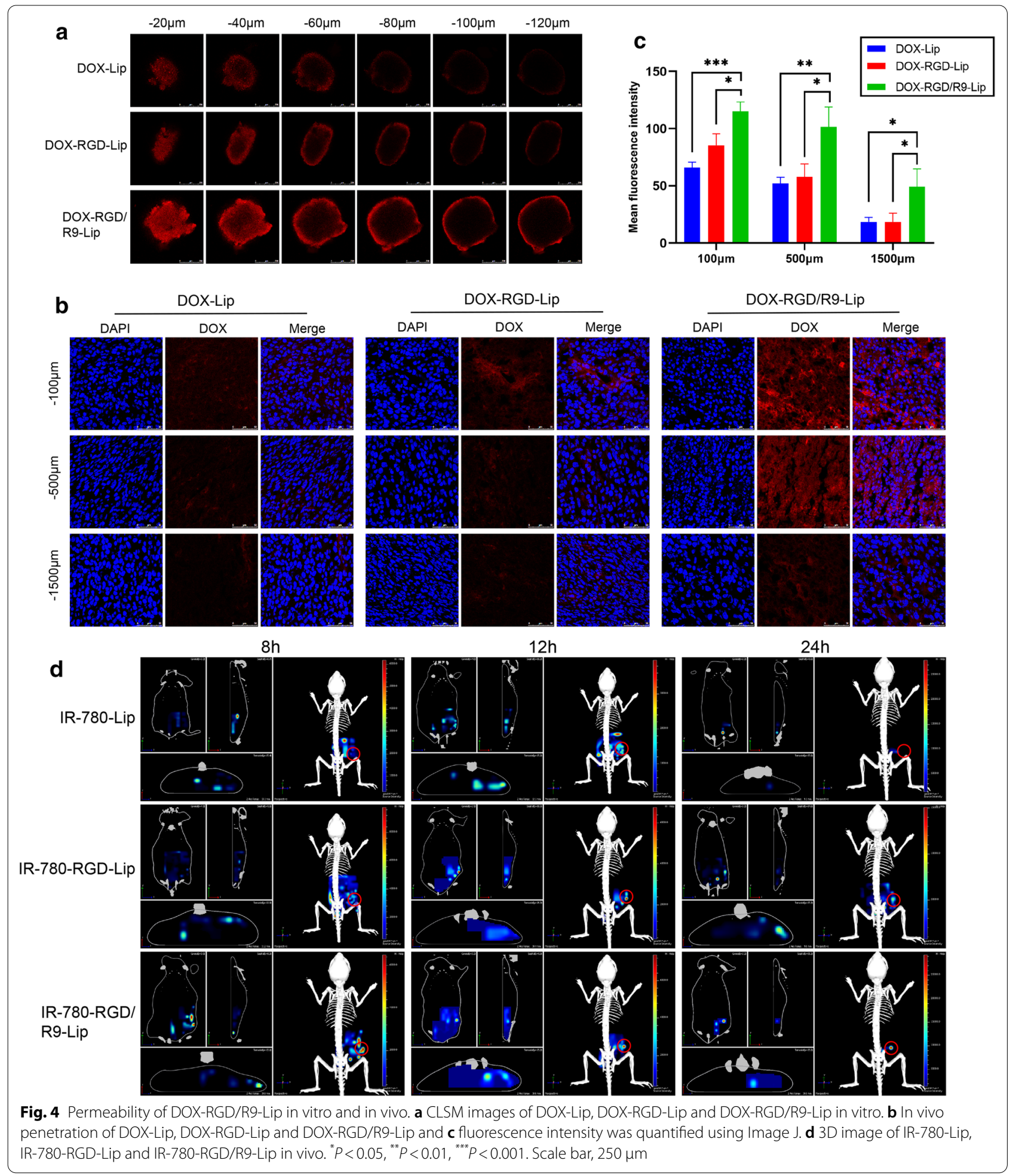

while all the liposomal groups showed increased fluorescent intensity, suggesting good long circulation of liposomes in colon cancer. Moreover, IR-780-RGD/
R9-sLip exhibited stronger fluorescence than both IR780-Lip and IR-780-RGD-Lip groups at all the timepoints, indicated that RGD/R9 modification would be 


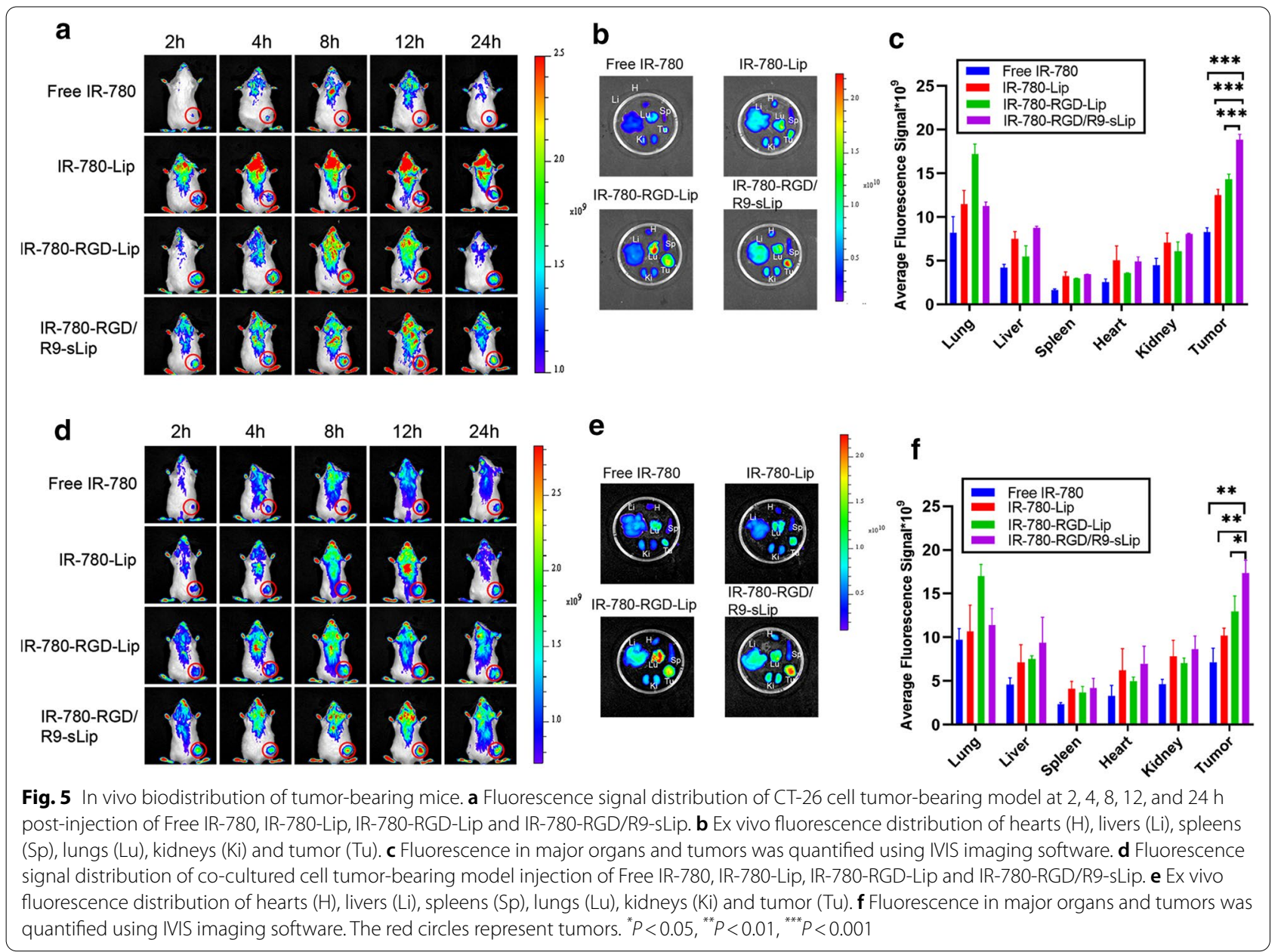

beneficial for improving the accumulation of the cargoes into tumor site.

\section{In vivo antitumor effect in CT-26 tumor-bearing mice model}

The antitumor effect of different formulations was evaluated in CT-26 bearing mice model. Compared with the saline group, all the other preparation groups showed different degrees of tumor growth inhibition (Fig. 6a-c). In addition, after treatment with IRI-RGD-Lip, the antitumor effect was further enhanced with tumor growth inhibition rate $56.63 \%$, which might be attributed to the increased cellular uptake via active targeting of RGD. Furthermore, the tumor inhibition rate of IRI-RGD/ R9-Lip group (68.88\%) was higher than that of the IRIRGD-Lip group $(P<0.05)$, which may be attributed to the deep penetration ability of R9. However, compared with IRI-RGD/R9-Lip group, no significant difference $(P>0.05)$ existed in IRI-RGD/R9-sLip, indicating that in the tumor-bearing model of single tumor cells, the addition of scFv had limited therapeutic effect duo to the absence of CAFs.

There was no significant difference in body weights among all treatment groups as shown in Fig. 6d. Besides, no visualized tissue damage was observed in H\&E section staining of major organs in mice, indicating that all preparations had no visible toxicities (Fig. 7). H\&E staining of the tumor sections was also performed to further evaluate the status on tumor growth. More cells lysis after treatment by IRI-RGD/R9-sLip demonstrated that tumor proliferation was significantly inhibited. Consistent with $H \& E$ results, apoptosis was most obvious in the TUNEL experiment and the number of Ki67 positive cells was reduced in the IRI-RGD/R9-sLip treatment group (Fig. 6e, f).

\section{In vivo antitumor effect in CT-26/NIH 3T3 tumor-bearing mice model}

In order to mimic the real CAFs-abundant tumor microenvironment, we further evaluated the antitumor effect in CT-26/NIH 3T3 co-cultured tumor-bearing mice 


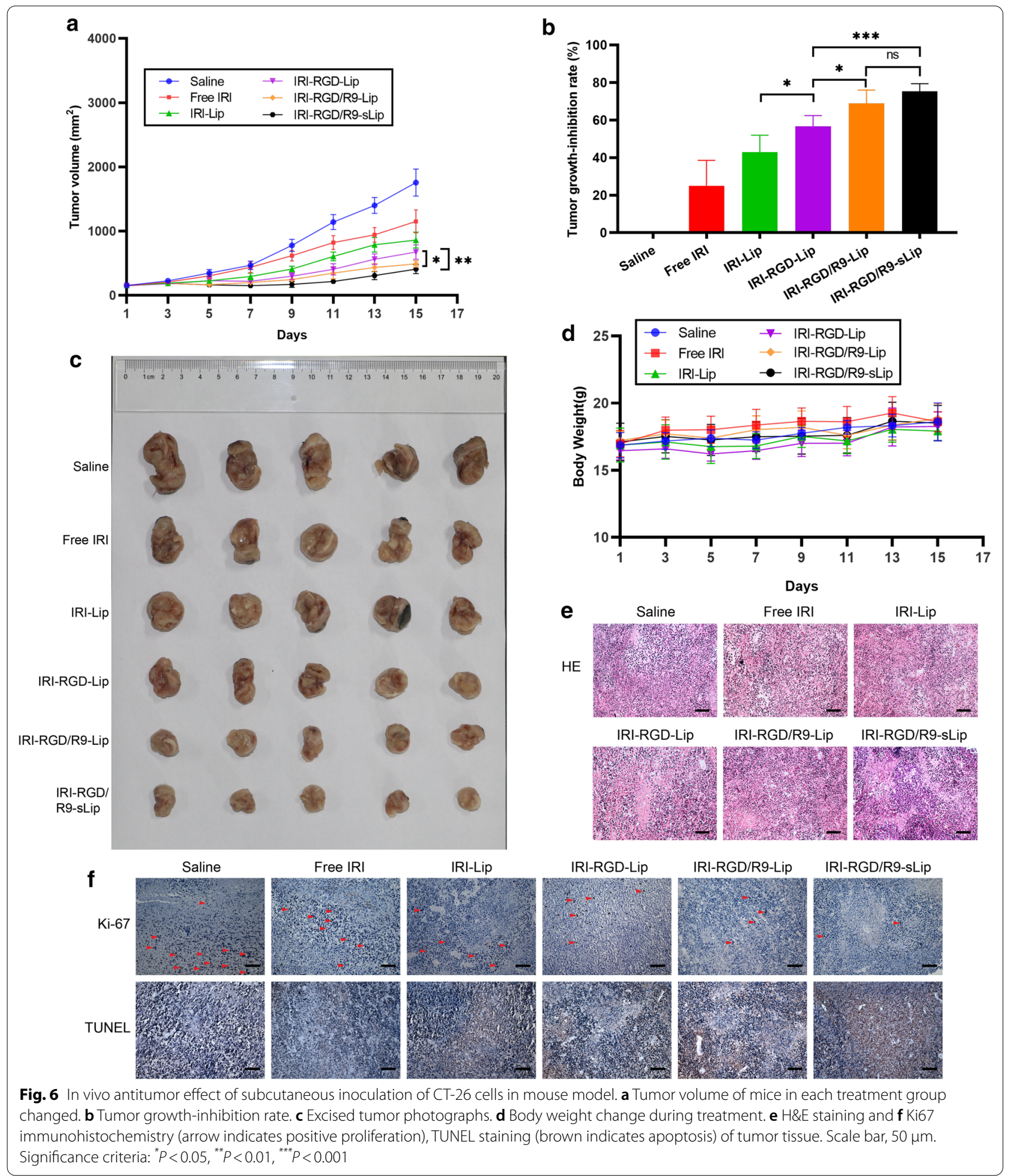

model. As shown in Fig. 8a, at the end of administration, tumor volumes in the saline group reached $\sim 3000 \mathrm{~mm}^{3}$, which was much higher than that in single CT-26 bearing mice model (about $2000 \mathrm{~mm}^{3}$ ). The faster tumor growth was attributed to the presence of a large number of stromal cells in the tumor microenvironment promoting the cell proliferation. IRI-RGD/R9-sLip exhibited superior anti-tumor advantages with the smallest tumor volumes 


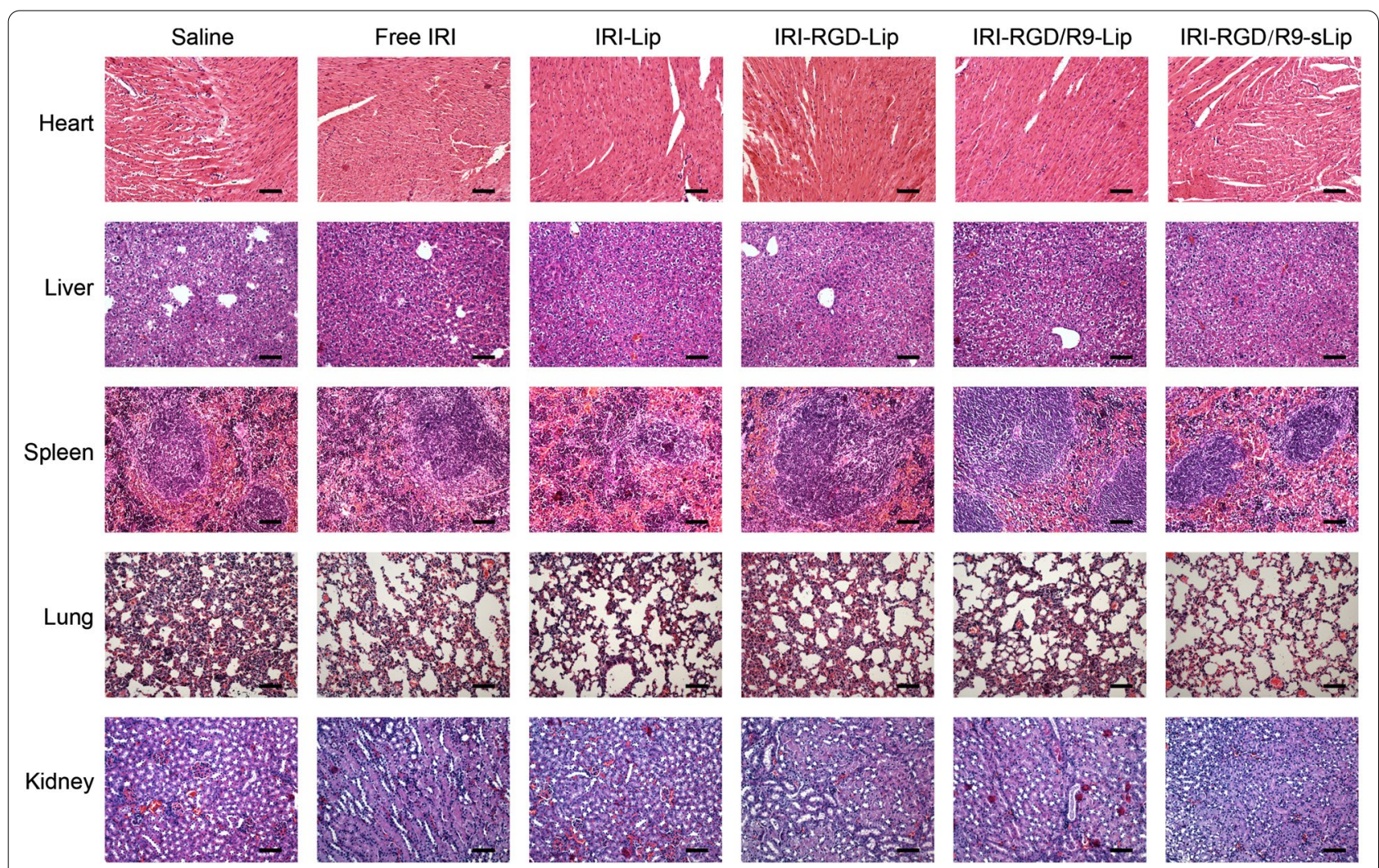

Fig. 7 The organs of mice in different treatment groups were stained by H\&E. Scale bar, $50 \mu \mathrm{m}$

compared to the other groups. Notably, the inhibition rate of IRI-RGD/R9-sLip group (70.97\%) was higher than that of IRI-RGD/R9-Lip (54.38\%) with significant difference (Fig. 8b), which was inconsistent with that in CT-26 bearing mice model. The results revealed that $\mathrm{scFv}$ could inhibit CAFs in the presence of a large number of CAFs, herein in favor of improving the antitumor effect. The tumor sizes also demonstrated that IRI-RGD/R9-sLip treatment could significantly inhibit the tumor growth (Fig. 8c). The above results suggested that in co-cultured tumor-bearing mice model, the combination of IRI and scFv could act on tumor cells and CAFs simultaneously to play a synergistic anti-tumor effect. Consequently, compared to the single tumor cells bearing mice model, co-culture cells would be a more reasonable model to reflect the real anti-tumor effect.

Moreover, immunohistochemistry on H\&E, TUNEL and Ki67 staining also showed the same results that the tumor sections in IRI-RGD/R9-sLip group had the most apoptotic and the least proliferative morphologies (Fig. 8e, f). The above results suggested that IRI combined with $\mathrm{scFv}$ has enhanced anti-tumor effect. Additionally, the expression of the biomarkers for CAFs including FAP and $\alpha$-SMA was further evaluated by immunohistochemistry. As shown in Fig. 9a and c, compared to CT-26 tumor-bearing mice, both FAP and $\alpha$-SMA expressions in CT-26/NIH 3T3 tumor-bearing mice were obviously increased, while significantly decreased after IRI-RGD/ R9-sLip treatment. According to the quantitative analysis in Fig. 9b and d, IRI-RGD/R9-sLip had lower expression of FAP and $\alpha$-SMA than the other groups, and there was significant difference compared to IRI-RGD/R9-Lip $(P<0.01)$. These results also verified that scFv could effectively kill CAFs and improve the anti-tumor effect of IRIRGD/R9-sLip. The in vivo function of scFv was firstly investigated, although single scFv could inhibit the tumor growth, the effect was insufficient with growth inhibition rate less than 40\% (Additional file 1: Fig. S8). In consideration of this, the combination of $\mathrm{scFv}$ and IRI would be more necessary, for which could co-act on both "seeds" and "soils" of the tumor, and thereby enhance the antitumor effect.

\section{In vivo antitumor effect in lung metastasis model and orthotopic tumor model}

The inhibition of tumor metastasis of different formulations was further studier by injecting CT-26 cells into tail vein of mice to establish lung metastasis model. As shown in the Fig. 10a, there was no significant weight loss during treatment, indicating that liposomes were provided with 


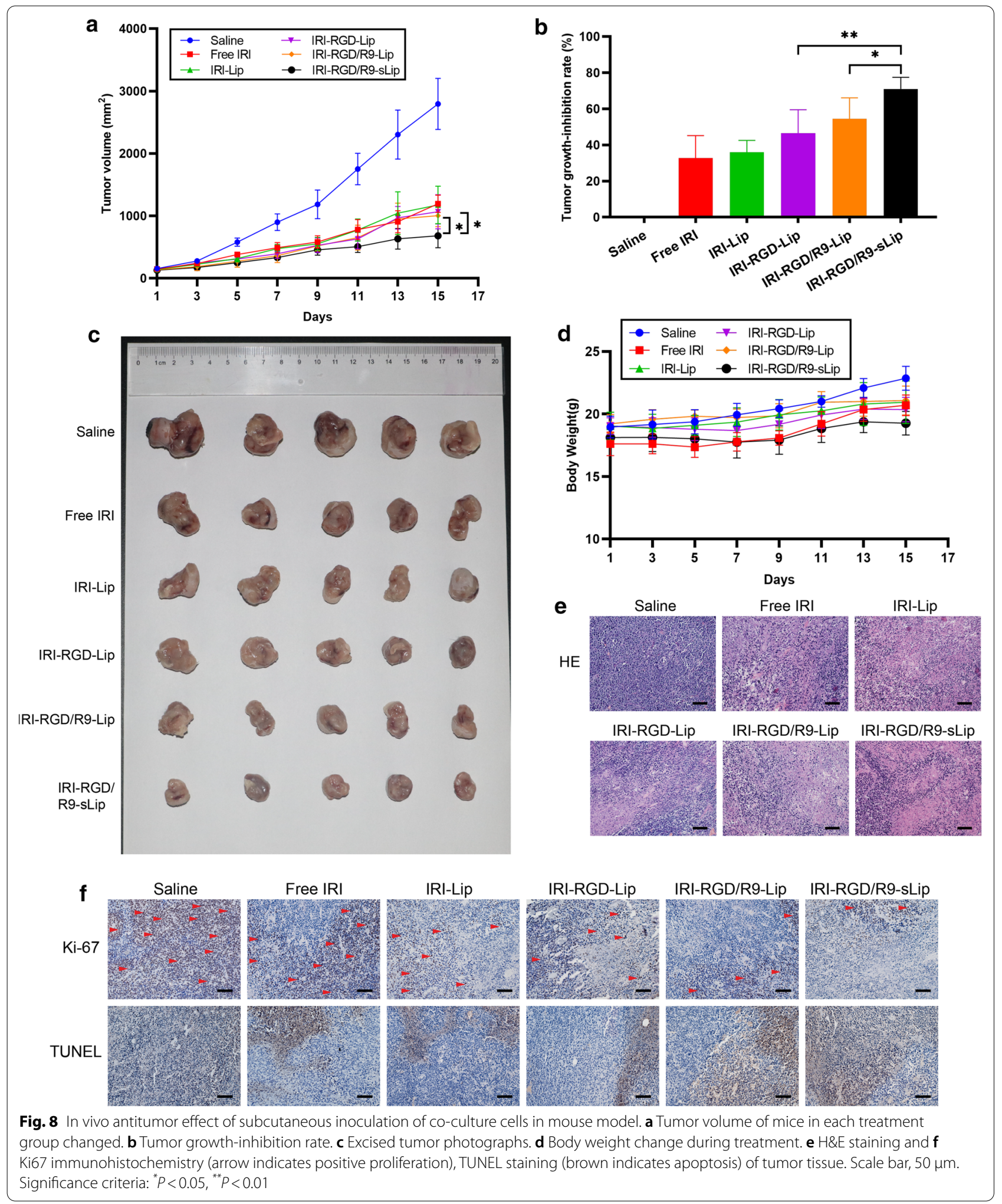



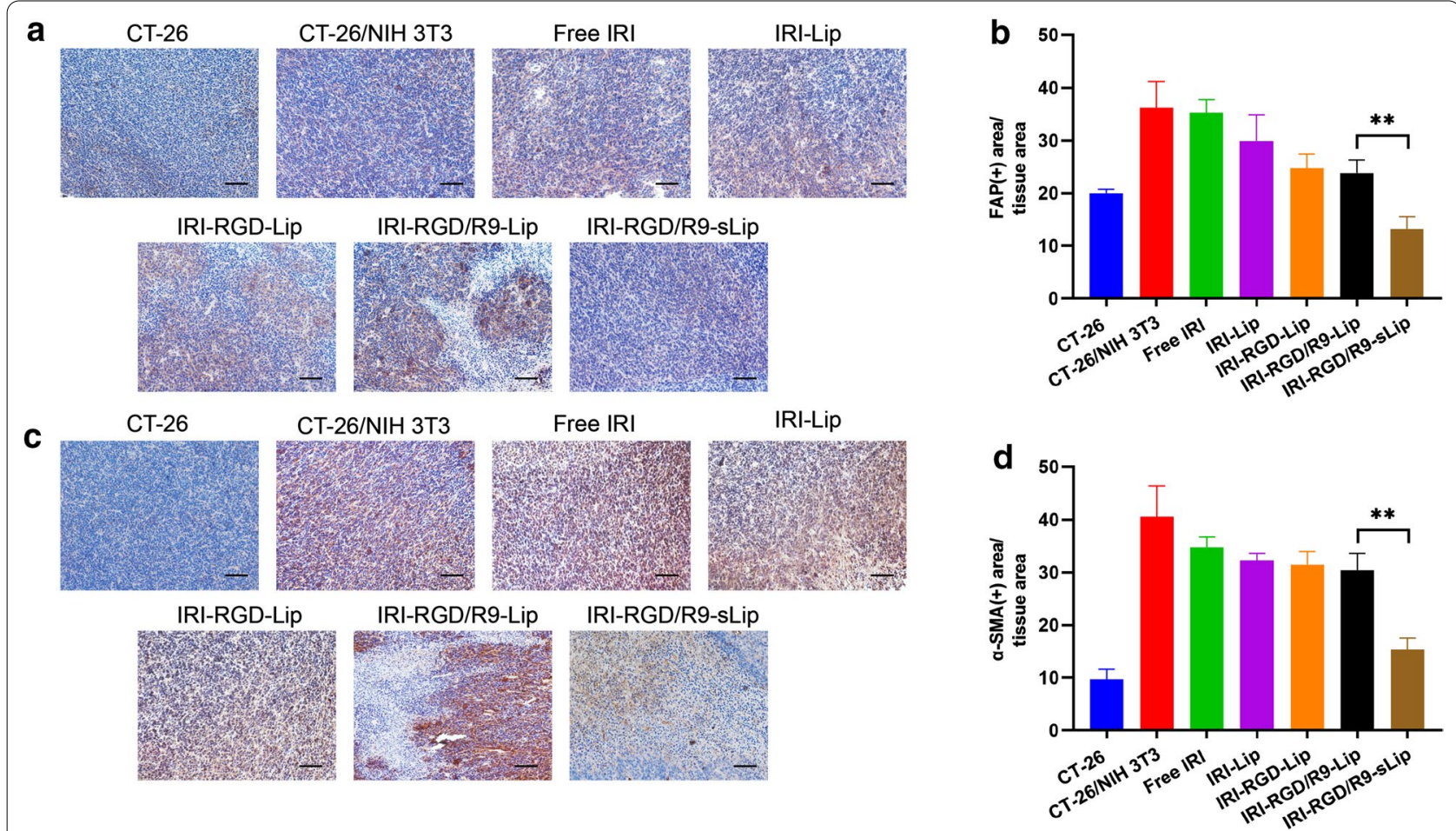

Fig. 9 Immunohistochemical analysis of tumor. a Qualitative images and $\mathbf{b}$ quantitative analysis of FAP. $\mathbf{c}$ Qualitative images and $\mathbf{d}$ quantitative analysis of a-SMA in the tumor. Scale bar, $50 \mu \mathrm{m}$. Significance criteria: ${ }^{* *} P<0.01$

satisfactory biocompatibility and could be safe for drug delivery. Excised lung images of mice in each group were shown in Fig. 10b and the number of nodules was further quantified in Fig. 10c. Compared to free IRI, metastatic nodules in IRI-RGD-Lip group were reduced, which was due to the improved targeting of RGD modification. IRIRGD/R9-sLip exhibited strongest anti-lung metastasis effect than the other groups $(P<0.01)$, which was attributed to the fact that IRI and $\mathrm{scFv}$ simultaneously killed tumor cells and CAFs. The histomorphology of lung was verified by H\&E staining. As shown in Fig. 10d, all the treatment groups had different degrees of abnormal pulmonary cell aggregation, while IRI-RGD/R9-sLip treatment group had small tumor lesions based on the combined treatment, indicating superior anti-tumor effect of IRI-RGD/R9-sLip. These results demonstrated our design that the co-loaded liposomes could simultaneously kill both tumor cells and CAFs, thus improving the anti-tumor effect and inhibiting the metastasis.

Furthermore, the orthotopic colon cancer model was established in order to truly evaluate the anti-CRC effect. As shown in Fig. 10(e), depauperate colon and fast-growing primary tumors with large volumes could be observed in the saline group, meanwhile, there were also more metastatic tumors on the small intestine wall. Compared to saline group, both free IRI and liposomal groups exhibited efficient therapeutic effect with smaller tumor volumes. Notably, smaller volumes of primary tumor and less metastatic tumors could be observed after IRI-RGD/R9-sLip treatment than free IRI. Consequently, it could be deduced that IRI-RGD/R9-sLip could significantly inhibit tumor growth and scFv could inhibit CAFs in orthotopic tumor model. Additionally, the colon tissues were further sectioned for H\&E staining (Fig. 10f). There was a larger area of tumor invasion in the saline group, while the tumor invasion site decreased after IRI treatment. In contrast, no obvious invasion was observed in IRI-RGD/R9-sLip group. These results suggested that IRI-RGD/R9-sLip could significantly improve the therapeutic effect.

\section{Conclusions}

In this study, the novel co-loaded multifunctional liposomes were developed that integrated multiple advantages, including IRI and scFv co-loading, scFv targeting and killing CAFs, RGD mediated active targeting, R9 improving the deep penetration of liposomes and lysosomal escape function, which could act on the tumor cells and destroy the "soil" of tumor cell survival simultaneously, and thus effectively reduce the metastasis and recurrence of malignant tumor. IRI-RGD/R9-sLip showed enhanced cytotoxicity in different cell models 


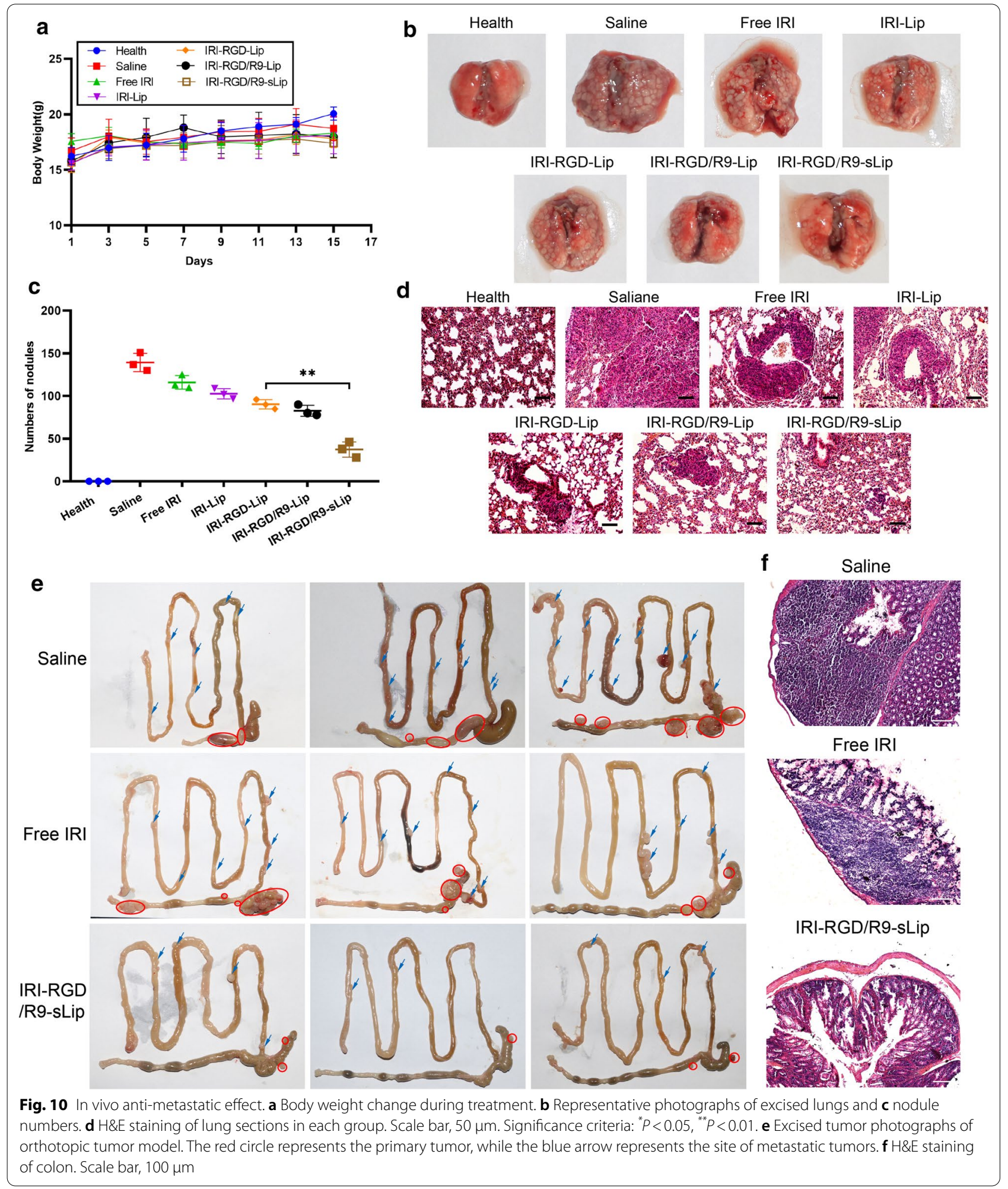

in vitro, effectively increased the accumulation in tumor sites in vivo and also exhibited deep permeation ability both in vitro and in vivo. Notably, IRI-RGD/R9-sLip not only exhibited superior in vivo anti-tumor effect in both
CAFs-free and CAFs-abundant bearing mice models, but also presented excellent anti-metastasis efficiency in lung metastasis model. In conclusion, the combinational strategy on killing both tumor cells and CAFs provides a new 
approach for cancer therapy, and the prepared co-loaded liposomes hold promising clinical application prospects.

\section{Supplementary Information}

The online version contains supplementary material available at https://doi. org/10.1186/s12951-021-01172-0.

Additional file 1: Table S1. Characterizations of DOX-loaded and IR780Liposomes. Fig. S1. 1H-NMR spectra characterization of (a) DSPE-PEG-RGD and (b) DSPE-PEG-R9. Fig. S2. Cell viability of (a) CT-26 cells or (b) cocultured cells (ratio of CT-26 to NIH 3T3 cells was 1:2) after incubation with blank liposome, RGD-Lip and RGD/R9-Lip at different concentrations for 48 h. Fig. S3. Cell viability of activated $\mathrm{NIH} 3 \mathrm{~T} 3$ cells after incubation with RGD/R9-Lip and RGD/R9-sLip for 48 h. Fig. S4. Cell viability of activated $\mathrm{NIH} 3 \mathrm{~T} 3$ cells after incubation with IRI for $48 \mathrm{~h}$. Fig. S5. The colocalization ratio of lysosome and liposome was quantified using Image J. *P $<0.05$, ${ }^{* *} P<0.01$. Fig. S6. The release profile of scFv after incubated with activated NIH3T3 cells. Fig. S7. In vivo biodistribution of orthotopic tumor model. (a) Fluorescence signal distribution of orthotopic tumor model at 2, 4, 8, 12, and 24 h post-injection of Free IR-780, IR-780-Lip, IR-780-RGDLip and IR-780-RGD/R9-sLip. (b) Ex vivo fluorescence distribution of hearts $(\mathrm{H})$, livers (Li), spleens (Sp), lungs (Lu), kidneys (Ki) and colon tumor (CT). Fig. S8. In vivo antitumor effect of subcutaneous inoculation of co-culture cells in mouse model. (a) Tumor volume of mice in each treatment group changed. (b) Tumor growth-inhibition rate. (c) Body weight change during treatment. (d) Excised tumor photographs. (e) Immunohistochemical analysis of tumor tissue. Scale bar, $50 \mu \mathrm{m}$.

Additional file 2. A representive video of 3D imaging in Fig. 4(d).

\section{Acknowledgements}

Not applicable.

\section{Authors' contributions}

ZHL and CXL contributed equally. ZHL, CXL and CLL designed experiments and co-wrote the paper. FQW and $\mathrm{JHL}$ contributed to some part of data analyses. ZJZ, JLW and BZ reviewed and edited the manuscript. All authors read and approved the final manuscript.

\section{Funding}

This work was financially supported by the National Science Foundation of China (No. 81803464) and Natural Science Foundations of Shandong Province (ZR2020MC078, ZR2018BH041). The work was also funded by the Domestic Visiting Scholar Program of Weifang Medical University (20207-02).

\section{Availability of data and materials}

All data generated or analyzed during this study are included in this published article.

\section{Declarations}

\section{Ethics approval and consent to participate}

All animal experiments were conducted in accordance with the Regulations on Animal Control issued by the Ministry of Health of the People's Republic of China and the Ethical Review of Animal Experiments issued by Weifang Medical University (2017-025).

\section{Consent for publication}

All authors agree to be published.

\section{Competing interests}

The authors declare that they have no competing interests.

\section{Author details}

'School of Pharmacy, Weifang Medical University Weifang, Shandong 261053, People's Republic of China. ${ }^{2}$ Department of Pharmacy, Qilu Hospital, Cheeloo College of Medicine, Shandong University, Ji'nan 250012, Shandong, People's
Republic of China. ${ }^{3}$ School of Bioscience and Technology, Weifang Medical University, Weifang 261053, Shandong, People's Republic of China.

Received: 29 April 2021 Accepted: 30 November 2021

Published online: 14 December 2021

\section{References}

1. Niu MK, Yi M, Dong B, Luo SX, Wu KM. Upregulation of STAT1-CCL5 axis is a biomarker of colon cancer and promotes the proliferation of colon cancer cells. Ann Transl Med. 2020;8(15):951.

2. Burtin F, Mullins CS, Linnebacher M. Mouse models of colorectal cancer: past, present and future perspectives. World J Gastroenterol. 2020;26(13):1394-426.

3. Juang V, Chang CH, Wang CS, Wang HE, Lo YL. pH-responsive PEGshedding and targeting peptide-modified nanoparticles for dual-delivery of irinotecan and microRNA to enhance tumor-specific therapy. Small. 2019;15(49):e1903296.

4. Bailly C. Irinotecan: 25 years of cancer treatment. Pharmacol Res. 2019;148:104398.

5. Weekes J, Lam AK, Sebesan S, Ho YH. Irinotecan therapy and molecular targets in colorectal cancer: a systemic review. World J Gastroenterol. 2009;15(29):3597-602.

6. Wulaningsih W, Wardhana A, Watkins J, Yoshuantari N, Repana D, Van Hemelrijck M. Irinotecan chemotherapy combined with fluoropyrimidines versus irinotecan alone for overall survival and progression-free survival in patients with advanced and/or metastatic colorectal cancer. Cochrane Database Syst Rev. 2016;2:CD008593.

7. Hui L, Chen Y. Tumor microenvironment: sanctuary of the devil. Cancer Lett. 2015;368(1):7-13.

8. Wang JJ, Lei KF, Han F. Tumor microenvironment: recent advances in various cancer treatments. Eur Rev Med Pharmaco. 2018;22(12):3855-64.

9. Quail DF, Joyce JA. Microenvironmental regulation of tumor progression and metastasis. Nat Med. 2013;19(11):1423-37.

10. Nunes SC. Tumor microenvironment-selective pressures boosting cancer progression. Adv Exp Med Biol. 2020;1219:35-49.

11. Li ZH, Zheng ZJ, Li CL, Li ZP, Wu JL, Zhang B. Therapeutic drugs and drug delivery systems targeting stromal cells for cancer therapy: a review. J Drug Target. 2020;28(7-8):714-26.

12. Ungefroren $H$, Sebens $S$, Seidl $D$, Lehnert $H$, Hass R. Interaction of tumor cells with the microenvironment. Cell Commun Signal. 2011;9:18.

13. Ren J, Ding L, Zhang D, Shi G, Xu Q, Shen S, Wang Y, Wang T, Hou Y. Carcinoma-associated fibroblasts promote the stemness and chemoresistance of colorectal cancer by transferring exosomal IncRNA H19. Theranostics. 2018;8(14):3932-48

14. Xouri G, Christian S. Origin and function of tumor stroma fibroblasts. Semin Cell Dev Biol. 2010;21(1):40-6.

15. Hintz HM, Cowan AE, Shapovalova M, LeBeau AM. Development of a cross-reactive monoclonal antibody for detecting the tumor stroma. Bioconjug Chem. 2019;30(5):1466-76.

16. Fang T, Lv H, Lv G, Li T, Wang C, Han Q, Yu L, Su B, Guo L, Huang S, Cao D, Tang L, Tang S, Wu M, Yang W, Wang H. Tumor-derived exosomal miR1247-3p induces cancer-associated fibroblast activation to foster lung metastasis of liver cancer. Nat Commun. 2018;9(1):191.

17. Kovacs D, Igaz N, Marton A, Ronavari A, Belteky P, Bodai L, Spengler G, Tiszlavicz L, Razga Z, Hegyi P, Vizler C, Boros IM, Konya Z, Kiricsi M. Core-shell nanoparticles suppress metastasis and modify the tumoursupportive activity of cancer-associated fibroblasts. J Nanobiotechnology. 2020;18(1):18.

18. Zhang J, Valianou M, Simmons H, Robinson MK, Lee HO, Mullins SR, Marasco WA, Adams GP, Weiner LM, Cheng JD. Identification of inhibitory scFv antibodies targeting fibroblast activation protein utilizing phage display functional screens. FASEB J. 2013;27(2):581-9.

19. Yang X, Lin Y, Shi Y, Li B, Liu W, Yin W, Dang Y, Chu Y, Fan J, He R. FAP promotes immunosuppression by cancer-associated fibroblasts in the tumor microenvironment via STAT3-CCL2 signaling. Cancer Res. 2016;76(14):4124-35.

20. de Sostoa J, Fajardo CA, Moreno R, Ramos MD, Farrera-Sal M, Alemany $R$. Targeting the tumor stroma with an oncolytic adenovirus secreting a 
fibroblast activation protein-targeted bispecific T-cell engager. J Immunother Cancer. 2019;7:1-15.

21. Rabenhold M, Steiniger F, Fahr A, Kontermann RE, Ruger R. Bispecific single-chain diabody-immunoliposomes targeting endoglin (CD105) and fibroblast activation protein (FAP) simultaneously. J Control Release. 2015;201:56-67.

22. Yu Q, Qiu Y, Li J, Tang X, Wang X, Cun X, Xu S, Liu Y, Li M, Zhang Z, He Q Targeting cancer-associated fibroblasts by dual-responsive lipid-albumin nanoparticles to enhance drug perfusion for pancreatic tumor therapy. J Control Release. 2020;321:564-75.

23. Fan F, Jin L, Yang L. pH-sensitive nanoparticles composed solely of membrane-disruptive macromolecules for treating pancreatic cancer. ACS Appl Mater Interfaces. 2021;13(11):12824-35.

24. Freedman JD, Duffy MR, Lei-Rossmann J, Muntzer A, Scott EM, Hagel J, Campo L, Bryant RJ, Verrill C, Lambert A, Miller P, Champion BR, Seymour LW, Fisher KD. An oncolytic virus expressing a T-cell engager simultaneously targets cancer and immunosuppressive stromal cells. Cancer Res. 2018;78(24):6852-65.

25. Ruger R, Tansi FL, Rabenhold M, Steiniger F, Kontermann RE, Fahr A, Hilger I. In vivo near-infrared fluorescence imaging of FAP-expressing tumors with activatable FAP-targeted, single-chain Fv-immunoliposomes. J Control Release. 2014;186:1-10.

26. Brocks B, Garin-Chesa P, Behrle E, Park JE, Rettig WJ, Pfizenmaier K, Moosmayer D. Species-crossreactive scFv against the tumor stroma marker "fibroblast activation protein" selected by phage display from an immunized FAP-/- knock-out mouse. Mol Med. 2001;7(7):461-9.

27. He C, Tang Z, Tian H, Chen X. Co-delivery of chemotherapeutics and proteins for synergistic therapy. Adv Drug Deliv Rev. 2016;98:64-76.

28. Hu TT, Cao H, Yang CL, Zhang LJ, Jiang XH, Gao X, Yang F, He G, Song XR, Tong AP, Gao G, Gong CY, Li R, Zhang XN, Wang XC, Zheng Y. LHDmodified mechanism-based liposome coencapsulation of mitoxantrone and prednisolone using novel lipid bilayer fusion for tissue-specific colocalization and synergistic antitumor effects. Acs Appl Mater Inter. 2016;8(10):6586-601.

29. Zhang L, Su H, Liu Y, Pang N, Li J, Qi XR. Enhancing solid tumor therapy with sequential delivery of dexamethasone and docetaxel engineered in a single carrier to overcome stromal resistance to drug delivery. J Control Release. 2019;294:1-16.

30. Yazdani S, Bansal R, Prakash J. Drug targeting to myofibroblasts: implications for fibrosis and cancer. Adv Drug Deliv Rev. 2017;121:101-16.

31. Li Y, Cheng Q, Jiang Q, Huang Y, Liu H, Zhao Y, Cao W, Ma G, Dai F, Liang X, Liang Z, Zhang X. Enhanced endosomal/lysosomal escape by distearoyl phosphoethanolamine-polycarboxybetaine lipid for systemic delivery of siRNA. J Control Release. 2014;176:104-14.

32. Varkouhi AK, Scholte M, Storm G, Haisma HJ. Endosomal escape pathways for delivery of biologicals. J Control Release. 2011;151(3):220-8.

33. Abu Lila AS, Ishida T. Liposomal delivery systems: design optimization and current applications. Biol Pharm Bull. 2017;40(1):1-10.

34. Sun D, Zhou JK, Zhao L, Zheng ZY, Li J, Pu W, Liu S, Liu XS, Liu SJ, Zheng $Y$, Zhao Y, Peng Y. Novel curcumin liposome modified with hyaluronan targeting CD44 plays an anti-leukemic role in acute myeloid leukemia in vitro and in vivo. ACS Appl Mater Interfaces. 2017;9(20):16857-68.

35. Li Y, Zhai Y, Liu W, Zhang K, Liu J, Shi J, Zhang Z. Ultrasmall nanostructured drug based $\mathrm{pH}$-sensitive liposome for effective treatment of drug-resistant tumor. J Nanobiotechnology. 2019;17(1):117.

36. Rubtsov MA, Syrkina MS, Aliev G. RGD-based therapy: principles of selectivity. Curr Pharm Des. 2016;22(7):932-52.

37. Hu H, Wang J, Wang H, Tan T, Li J, Wang Z, Sun K, Li Y, Zhang Z. Cellpenetrating peptide-based nanovehicles potentiate lymph metastasis targeting and deep penetration for anti-metastasis therapy. Theranostics. 2018;8(13):3597-610.

38. Guo F, Fu Q, Zhou K, Jin C, Wu W, Ji X, Yan Q, Yang Q, Wu D, Li A, Yang G. Matrix metalloprotein-triggered, cell penetrating peptide-modified star-shaped nanoparticles for tumor targeting and cancer therapy. J Nanobiotechnology. 2020;18(1):48.

39. Ji T, Ding Y, Zhao Y, Wang J, Qin H, Liu X, Lang J, Zhao R, Zhang Y, Shi J, Tao N, Qin Z, Nie G. Peptide assembly integration of fibroblast-targeting and cell-penetration features for enhanced antitumor drug delivery. Adv Mater. 2015;27(11):1865-73.
40. Maniti O, Piao HR, Ayala-Sanmartin J. Basic cell penetrating peptides induce plasma membrane positive curvature, lipid domain separation and protein redistribution. Int J Biochem Cell Biol. 2014;50:73-81.

41. Shi NQ, Li Y, Zhang Y, Shen N, Qi L, Wang SR, Qi XR. Intelligent "PeptideGathering Mechanical Arm"Tames Wild "Trojan-Horse" peptides for the controlled delivery of cancer nanotherapeutics. ACS Appl Mater Interfaces. 2017:9(48):41767-81.

42. Li C, Guan H, Li Z, Wang F, Wu J, Zhang B. Study on different particle sizes of DOX-loaded mixed micelles for cancer therapy. Colloids Surf B Biointerfaces. 2020;196:111303.

43. Fan Y, Wang QJ, Lin GM, Shi YB, Gu ZL, Ding TT. Combination of using prodrug-modified cationic liposome nanocomplexes and a potentiating strategy via targeted co-delivery of gemcitabine and docetaxel for CD44-overexpressed triple negative breast cancer therapy. Acta Biomater. 2017;62:257-72.

44. Li ZA, Zhang JJ, Zhou JW, Lu LL, Wang HS, Zhang G, Wan GH, Cai SH, Du J. Nodal facilitates differentiation of fibroblasts to cancer-associated fibroblasts that support tumor growth in melanoma and colorectal cancer. Cells-Basel. 2019;8(6):538.

45. Zhu D, Yan H, Liu X, Xiang J, Zhou Z, Tang J, Liu X, Shen Y. Intracellularly disintegratable polysulfoniums for efficient gene delivery. Adv Func Mater. 2017;27(16):1606826.

46. Zhu D, Yan H, Zhou Z, Tang J, Liu X, Hartmann R, Parak WJ, Shen Y, Feliu N. Influence of the modulation of the protein corona on gene expression using polyethylenimine (PEI) polyplexes as delivery vehicle. Adv Healthc Mater. 2021;10(13):e2100125.

47. Wang C, Zhao M, Liu YR, Luan X, Guan YY, Lu Q, Yu DH, Bai F, Chen HZ, Fang C. Suppression of colorectal cancer subcutaneous xenograft and experimental lung metastasis using nanoparticle-mediated drug delivery to tumor neovasculature. Biomaterials. 2014;35(4):1215-26.

48. Wei C, Yang C, Wang S, Shi D, Zhang C, Lin X, Liu Q, Dou R, Xiong B. Crosstalk between cancer cells and tumor associated macrophages is required for mesenchymal circulating tumor cell-mediated colorectal cancer metastasis. Mol Cancer. 2019;18(1):64.

49. Zeng Z, Li Y, Pan Y, Lan X, Song F, Sun J, Zhou K, Liu X, Ren X, Wang F, Hu J, Zhu X, Yang W, Liao W, Li G, Ding Y, Liang L. Cancer-derived exosomal miR-25-3p promotes pre-metastatic niche formation by inducing vascular permeability and angiogenesis. Nat Commun. 2018;9(1):5395.

50. Han YH, Mun JG, Jeon HD, Kee JY, Hong SH. Betulin inhibits lung metastasis by inducing cell cycle arrest, autophagy, and apoptosis of metastatic colorectal cancer cells. Nutrients. 2019;12(1):66.

51. Jiang M, Li W, Zhu C, Li X, Zhang J, Luo Z, Qin B, Du Y, Luo L, You J. Perdurable PD-1 blockage awakes anti-tumor immunity suppressed by precise chemotherapy. J Control Release. 2021;329:1023-36.

52. Li Y, Wang C, Li D, Deng P, Shao X, Hu J, Liu C, Jie H, Lin Y, Li Z, Qian X, Zhang H, Zhao Y. 1H-NMR-based metabolic profiling of a colorectal cancer CT-26 lung metastasis model in mice. Oncol Rep. 2017;38(5):3044-54.

\section{Publisher's Note}

Springer Nature remains neutral with regard to jurisdictional claims in published maps and institutional affiliations.

Ready to submit your research? Choose BMC and benefit from:

- fast, convenient online submission

- thorough peer review by experienced researchers in your field

- rapid publication on acceptance

- support for research data, including large and complex data types

- gold Open Access which fosters wider collaboration and increased citations

- maximum visibility for your research: over $100 \mathrm{M}$ website views per year

At BMC, research is always in progress.

Learn more biomedcentral.com/submissions 